\title{
A Hamilton-Jacobi point of view on mean-field Gibbs-non-Gibbs transitions
}

\author{
Richard C. Kraaij ${ }^{\mathrm{I}}$ \\ Frank Redig II \\ Willem B. van Zuijlen III
}

February 9, 2021

\begin{abstract}
We study the loss, recovery, and preservation of differentiability of time-dependent large deviation rate functions. This study is motivated by mean-field Gibbs-nonGibbs transitions.

The gradient of the rate-function evolves according to a Hamiltonian flow. This Hamiltonian flow is used to analyze the regularity of the time-dependent rate function, both for Glauber dynamics for the Curie-Weiss model and Brownian dynamics in a potential. We extend the variational approach to this problem of time-dependent regularity in order to include Hamiltonian trajectories with a finite lifetime in closed domains with a boundary. This leads to new phenomena, such a recovery of smoothness.

We hereby create a new and unifying approach for the study of mean-field Gibbsnon-Gibbs transitions, based on Hamiltonian dynamics and viscosity solutions of Hamilton-Jacobi equations.
\end{abstract}

AMS 2010 subject classification: 49L99, 60F10, 82C22, 82C27.

Keywords: Hamiltonian dynamics, Hamilton-Jacobi equation, Mean-field models, large deviation principle, Gibbs versus non-Gibbs, dynamical transition, global minimisers of rate functions

\section{Introduction}

The large deviation approach to dynamical Gibbs-non-Gibbs transitions, initiated in van Enter, Fernández, den Hollander and Redig [vEFdHR10], characterizes the emergence of 'bad configurations' via the non-uniqueness of optimal starting configurations corresponding to a given arrival configuration. 'Bad configurations' have to be interpreted

\footnotetext{
${ }^{\mathrm{I} D e l f t}$ Institute of Applied Mathematics, Technische Universiteit Delft, Delft, the Netherlands, r.c.kraaij@tudelft.nl.

${ }^{\mathrm{II}}$ Delft Institute of Applied Mathematics, Technische Universiteit Delft, Delft, the Netherlands, f.h.j.redig@tudelft.nl.

${ }^{\text {III } W e i e r s t r a s s ~ I n s t i t u t e, ~ B e r l i n, ~ G e r m a n y, ~ v a n z u i j l e n @ w i a s-b e r l i n . d e . ~}$
} 
as points of essential discontinuity of conditional probabilities and 'optimal' has to be interpreted here in the sense of minimizing a large deviation cost,

$$
I(\gamma)=I_{0}(\gamma(0))+\int_{0}^{\infty} \mathscr{L}(\gamma(s), \dot{\gamma}(s)) \mathrm{d} s,
$$

which is the sum of an initial cost $I_{0}$ corresponding to the starting measure and a pathspace cost in the form of a Lagrangian action. In the mean-field context one considers trajectories of the magnetization and the dynamical Gibbs-non-Gibbs transitions are to be interpreted in the sense of Gibbsianness for mean-field models, a notion introduced in Külske and le Ny [KLN07] and studied in EK10, FdHM13, dHRvZ15, KK19, KM20]. Inspired from the literature on dynamical Gibbs-non-Gibbs transitions it is natural to conjecture that for a large variety of models the following three statements are equivalent:

(a) Mean-field Gibbsianness at time $t$;

(b) Unique optimal trajectories: for all arrival points $b$ at time $t$ the optimal trajectory arriving at $b$ is unique, i.e.,

$$
\underset{\gamma, \gamma(t)=b}{\operatorname{argmin}} I_{0}(\gamma(0))+\int_{0}^{t} \mathscr{L}(\gamma(s), \dot{\gamma}(s)) \mathrm{d} s
$$

is a singleton;

(c) Differentiability of the rate function at time $t$, i.e., $I_{t}$ given by

$$
I_{t}(b):=\inf _{\gamma, \gamma(t)=b} I_{0}(\gamma(0))+\int_{0}^{t} \mathscr{L}(\gamma(s), \dot{\gamma}(s)) \mathrm{d} s,
$$

is differentiable as a function of $b$.

In this paper, we prove, for a broad class of models in the one-dimensional setting, the equivalence of (b) and (c) and introduce new methods within the framework of calculus of variations to investigate the differentiability of the rate function at time $t$. The proofs are based on techniques from the theory of calculus of variations. Under certain conditions the equivalence of (b) and (c) has been proved, however, our setting is fundamentally different as will be explained below.

By using this general approach, we do not use specific information of the considered models and therefore our methods are applicable in a large variety of models. This is in contrast to [KLN07, EK10, FdHM13, dHRvZ15, KK19, KM20], whose approaches rely on explicit information about the specific models, for which they treat the relation between (a) and (b) .

Let $H(x, p)=\sup _{v} p v-\mathscr{L}(x, v)$ be the Hamiltonian corresponding to the Lagrangian in (11). Following classical mechanics, if the characteristics of the Hamilton-Jacobi equation

$$
\partial_{t} u(t, x)+H\left(x, \partial_{x} u(t, x)\right)=0, \quad u(0, x)=I_{0}(x),
$$

do not intersect, then $u(t, x):=I_{t}(x)$, with $I_{t}$ defined in (2), is continuously differentiable and a classical solution of (3). Following the theory of calculus of variations, even if the 
characteristics intersect, then $u(t, x)=I_{t}(x)$ still solves (3) as a viscosity solution. In addition, one can show that $u$ is locally semi-concave.

The characteristics of (3) are exactly the Hamilton trajectories, i.e., they solve the Hamilton equations

$$
\left[\begin{array}{c}
\dot{X}(s) \\
\dot{P}(s)
\end{array}\right]=\left[\begin{array}{c}
\partial_{p} H(X(s), P(s)) \\
-\partial_{x} H(X(s), P(s))
\end{array}\right] .
$$

Moreover, every optimal trajectory as in (b) above has an associated characteristic. A rigorous analysis shows that these observations can be turned into a proof that (b) is equivalent to (c). In addition, a study of solutions to (4) can be used to prove or disprove that $I_{t}$ is differentiable.

We proceed with giving three techniques based on the analysis of Hamiltonian flows that either guarantee differentiability or non-differentiability of $I_{t}$ : order preservingness, linearization and rotation. We illustrate these methods on the natural examples of Glauber dynamics for the Curie-Weiss model and that of mean-field interacting Brownian particles in a single- or double-well potential. Phenomena such as short-time conservation, loss and recovery of Gibbsianness (differentiability) for both high- and lowtemperature dynamics, previously obtained in specific examples only, are now obtained in a broad context.

Comparison with existing literature on calculus of variations. One of the earlier works dealing with the equivalence between (b) and (c) is Fle69. The theory of viscosity solutions, their regularity and Hamiltonian trajectories is a well established theory, we refer to the works [Lio82], [FS89], [LY95], [BC97], [CS04] and [FS06]. However, these works do not apply in our context. Indeed, in the references mentioned above the spaces are open and all Hamilton trajectories have an infinite life time (they stay at all times in the open set) and the Lagrangian is finite and continuously differentiable on the closure of the space. A major difficulty that we have to overcome is that the mean-field models which we consider have Hamiltonians for which the solutions to (4) have a finite time of existence, after which they arrive at 'points at infinity'. Also the Lagrangian is not finite on the boundary of the space. These problems are not merely technical: they also lead to new phenomena, such as 'recovery of differentiability' which in the Gibbs-non-Gibbs literature corresponds to 'recovery of Gibbsianness' (which for measures on the lattice has been shown in vEFdHR02 and for infinite-temperature mean-field dynamics in [FdHM13] $)$.

Overview. The rest of our paper is organized as follows.

In Section 2 we introduce Gibbs-non-Gibbs transitions, path-space large deviations, examples of models giving rise to Hamiltonians that fall within our framework and some definitions and preleminaries from the theory of calculus of variations. Then we give our main result on the equivalence between (b) and (c), the relation between uniqueness of optimisers and the regularity of the rate function and finally we present a relation between this regularity and the push-forward of the graph of the derivative of the initial rate function.

In Section 3 we prescribe conditions under which the regularity is preserved or broken 
and apply this to obtain different scenarios for the models introduced in Section 2 . One of the scenarios treated here it the one of recovery, as mentioned before.

Techniques and proofs for theorems of Section 2 can be found in Section 4 , 5 and 6 .

Techniques and proofs for theorems of Section 3 can be found in Section 7,8 and 9 .

Acknowledgements. The authors are grateful to C. Külske and A.C.M. van Rooij for discussions.

RK is supported by The Netherlands Organisation for Scientific Research (NWO), grant number 600.065.130.12N109 and the Deutsche Forschungsgemeinschaft (DFG) via RTG 2131 High-dimensional Phenomena in Probability - Fluctuations and Discontinuity.

WvZ is supported by the German Science Foundation (DFG) via the Forschergruppe FOR2402 "Rough paths, stochastic partial differential equations and related topics".

\section{Preliminaries and theoretical main results}

\subsection{Mean-field Gibbs measures}

In this paper, as the initial model at time $t=0$ we consider two mean-field models. In the next two sections we will describe the dynamics to which these initial models will be subjected. Namely, we consider one with spins that attain values in $\mathbb{R}$, which we refer to as the $\mathbb{R}$-space-model, and one with spins that attain values in $\{-1,1\}$, which we refer to as the \pm 1 -space-model. We will write $\mathbb{K}$ for the space in which empirical averages will take their values, in particular we have that $\mathbb{K}$ equals $\mathbb{R}$ for the $\mathbb{R}$-space-model, and $[-1,1]$ for the \pm 1 -space-model.

We start in both cases from an initial measure $\mu_{N, 0}$ of the form

$$
\mu_{N, 0}\left(\mathrm{~d} \sigma_{1}, \cdots, \mathrm{d} \sigma_{N}\right)=\frac{e^{-N V\left(m_{N}\left(\sigma_{1}, \ldots, \sigma_{N}\right)\right)}}{Z_{N}} \lambda^{N}\left(\mathrm{~d} \sigma_{1}, \cdots, \mathrm{d} \sigma_{N}\right),
$$

where

$$
m_{N}\left(\sigma_{1}, \ldots, \sigma_{N}\right)=\frac{1}{N} \sum_{i=1}^{N} \sigma_{i}
$$

$\lambda^{N}$ is the $N$-fold product of $\lambda, Z_{N}$ the normalizing constant, and

(i) for the $\mathbb{R}$-space-model, $V: \mathbb{R} \rightarrow[0, \infty)$ is continuous and $\lambda$ is a standard normal distribution on $\mathbb{R}$.

(ii) for the \pm 1 -space-model, $V:[-1,1] \rightarrow \mathbb{R}$ is continuous and $\lambda$ is the uniform measure on $\{-1,1\}$.

The "potential" $V$ determines in both cases uniquely the rate function for the large deviation principle of the magnetization $m_{N}$ under $\mu_{N, 0}$, which is the function

$$
x \mapsto V(x)+i(x)-\inf _{x \in \mathbb{K}}(V(x)+i(x)),
$$

where 
(i) for the $\mathbb{R}$-space-model, $i(x)=\frac{1}{2} x^{2}$,

(ii) for the \pm 1 -space-model, $i(x)=\frac{1-x}{2} \log (1-x)+\frac{1+x}{2} \log (1+x)$.

We consider the spins to evolve according to the following dynamics

(i) for the $\mathbb{R}$-space-model; interacting diffusions as described in Section 2.3 .

(ii) for the \pm 1 -space-model; Glauber dynamics as described in Section 2.2 .

The initial measure $\mu_{N, 0}$ is transformed by the dynamics to the measure $\mu_{N, t}$ at time $t>0$.

Definition 2.1. Let $t \geq 0 . \alpha \in \mathbb{K}$ is called a good magnetization for $\left(\mu_{N, t}\right)_{N \in \mathbb{N}}$ if there exists a probability measure $\gamma_{t}(\cdot \mid \alpha)$ such that

$$
\mu_{N, t}\left(\mathrm{~d} \sigma_{1} \mid \sigma_{2}^{N}, \ldots, \sigma_{N}^{N}\right) \stackrel{\text { weakly }}{\longrightarrow} \gamma_{t}\left(\mathrm{~d} \sigma_{1} \mid \alpha\right),
$$

for all $\sigma_{2}^{N}, \ldots, \sigma_{N}^{N}$ such that $m_{N-1}\left(\sigma_{2}^{N}, \ldots, \sigma_{N}^{N}\right) \rightarrow \alpha$. If $\alpha$ is not a good magnetization, it is called a bad magnetization.

The sequence $\left(\mu_{N, t}\right)_{N \in \mathbb{N}}$ is called sequentially Gibbs if $\alpha$ is a good magnetisation for all $\alpha \in \mathbb{K}$.

If $\left(\mu_{n, t}\right)_{N \in \mathbb{N}}$ is sequentially Gibbs, then $\alpha \mapsto \gamma_{t}(\cdot \mid \alpha)$ is weakly continuous (see vZ16, Theorem 3.A.1] or dHRvZ15, Lemma 1.3]).

Remark 2.2. The definition of sequentially Gibbs follows those in [KLN07, [EK10], FdHM13], dHRvZ15. We refer to [KLN07] for the explanation of the definition with regard to Gibbs measures on the lattice.

\subsection{Glauber dynamics}

In this section, we describe the dynamics for the \pm 1 -space-model. For each $N$, we consider a continuous-time Markov process $\left(X_{1}(t), \cdots, X_{N}(t)\right) \in\{-1,1\}^{N}$ of mean-field interacting spins with mean-field jump rates $c_{N}$. The law of $\left(X_{1}(0), \cdots, X_{N}(0)\right)$ is $\mu_{N, 0}$ and the Markov generators of these spin-flip systems are of the form

$$
\mathscr{A}_{N} f\left(\sigma_{1}, \ldots, \sigma_{N}\right):=\sum_{i=1}^{N} c_{N}\left(\sigma_{i}, m_{N}(\sigma)\right)\left[f\left(\sigma^{i}\right)-f(\sigma)\right],
$$

where $c_{N} \geq 0$ and where the configuration $\sigma^{i} \in\{-1,1\}^{N}$ is given by

$$
\sigma_{j}^{i}= \begin{cases}-\sigma_{j} & \text { if } i=j \\ \sigma_{j} & \text { if } i \neq j\end{cases}
$$

We denote by $M_{N}(t):=\frac{1}{N} \sum_{i=1}^{N} X_{i}(t)$ the empirical magnetization at time $t$. Due to the mean-field character of this dynamics, also the dynamics of the empirical magnetization is Markovian, and an elementary computation shows that the generator of the

\footnotetext{
${ }^{1}$ In case $\mathbb{K}=\mathbb{R}$, the conditional measure on the left-hand-side of $(8)$ has to be understood in terms of weakly continuous regular conditional probabilities as is done in dHRvZ15.
} 
process $\left(M_{N}(t)\right)_{t \geq 0}$ on $m_{N}\left(\{-1,1\}^{N}\right) \subseteq[-1,1]$ is given by

$$
\begin{aligned}
A_{N} f(x)= & N \frac{1-x}{2} c_{N}(-1, x)\left[f\left(x+2 N^{-1}\right)-f(x)\right] \\
& +N \frac{1+x}{2} c_{N}(+1, x)\left[f\left(x-2 N^{-1}\right)-f(x)\right],
\end{aligned}
$$

as it satisfies $\mathscr{A}_{N}\left(f \circ m_{N}\right)=\left(A_{N} f\right) \circ m_{N}$. For later purposes, we assume the following.

Assumption 2.3. There exist functions $v_{+}, v_{-}:[-1,1] \rightarrow[0, \infty)$ such that

$$
\lim _{N \rightarrow \infty} \sup _{x \in m_{N}\left(\{-1,1\}^{N}\right)}\left|\frac{1-x}{2} c_{N}(-1, x)-v_{+}(x)\right|+\left|\frac{1+x}{2} c_{N}(+1, x)-v_{-}(x)\right|=0,
$$

for which the following properties hold:

(a) $v_{-}(-1)=0, v_{-}(x)>0$ for $x \neq 1$, and $v_{+}(1)=0$ and $v_{+}(x)>0$ for $x \neq 1$,

(b) $v_{-}, v_{+}$have an extensions to an open set $V \subseteq \mathbb{R}$ that contains $[-1,1]$ and these extensions are twice continuously differentiable,

(c) $v_{+}^{\prime}(1)<0$ and $v_{-}^{\prime}(-1)>0$.

In concrete examples, we consider $v_{-}, v_{+}$of the form

$$
v_{-}(x)=\frac{1+x}{2} e^{-\beta x-h}, \quad v_{+}(x)=\frac{1-x}{2} e^{\beta x+h},
$$

which correspond to the rates obtained from Glauber spin-flip dynamics reversible with respect to the Curie-Weiss measure in (5) at inverse temperature $\beta \geq 0$ and external magnetic field $h \in \mathbb{R}$, i.e., for $V(x)=-\beta x^{2}-h x$.

\subsection{Interacting diffusion processes}

In this section, we describe the dynamics for the $\mathbb{R}$-space-model. For each $N$, we consider $N$ mean-field interacting diffusions $\left(X_{1}(t), \ldots, X_{N}(t)\right) \in \mathbb{R}^{N}$ in a potential landscape $W_{N}: \mathbb{R} \rightarrow \mathbb{R}$, where $W_{N}$ is continuously differentiable. We assume that $-W_{N}^{\prime}$ is onesided Lipschitz: there is some $M \geq 0$ such that for all $x>y$

$$
-\left(W_{N}^{\prime}(x)-W_{N}^{\prime}(y)\right) \leq M(x-y) .
$$

The law of $\left(X_{1}(0), \ldots, X_{N}(0)\right)$ is given by $\mu_{N, 0}$ and the dynamics are given by

$$
\mathrm{d} X_{i}(t)=-W_{N}^{\prime}\left(M_{N}(t)\right) \mathrm{d} t+\mathrm{d} B_{i}(t)
$$

where $M_{N}(t):=\frac{1}{N} \sum_{i=1}^{N} X_{i}(t)$ is the empirical magnetization at time $t$ as above and where $B_{1}, \ldots, B_{N}$ are independent standard Brownian motions. Note that there exists a unique solution to this stochastic differential equation by [PR14, Proposition 3.38] and the one-sided Lipschitz property of $-W_{N}^{\prime}$. The empirical magnetization is also Markovian and satisfies

$$
\mathrm{d} M_{N}(t)=-W_{N}^{\prime}\left(M_{N}(t)\right) \mathrm{d} t+\frac{1}{\sqrt{N}} \mathrm{~d} B_{1}(t) .
$$


Again by [PR14, Proposition 3.38] this equation has a unique solution, and additionally, its generator $A_{N}$ with domain $C_{b}^{2}(\mathbb{R})$ is given by

$$
A_{N} f(x)=-W_{N}^{\prime}(x) f^{\prime}(x)+\frac{1}{2 N} f^{\prime \prime}(x) .
$$

Also in this case we have the following assumption.

Assumption 2.4. We assume that there is some three times continuously differentiable function $W: \mathbb{R} \rightarrow \mathbb{R}$ for which $-W^{\prime}$ is one-sided Lipschitz and such that for every compact set $K \subseteq \mathbb{R}$

$$
\lim _{N \rightarrow \infty} \sup _{x \in K}\left|W_{N}^{\prime}(x)-W^{\prime}(x)\right|=0 .
$$

$W(x)=\sum_{i=1}^{2 k} a_{i} x^{i}$ with $a_{i} \in \mathbb{R}$ and $a_{2 k}>0$ is an example of such a function for which $-W^{\prime}$ is one-sided Lipschitz. In the examples that we will consider, we will use $W(x)=\frac{1}{4} x^{4}-\frac{1}{2} d x^{2}$ with $d \in \mathbb{R}$. This function is strictly convex for $d \leq 0$ ('high temperature') and has the shape of a double well for $d>0$ ('low temperature').

\subsection{Path-space large deviations}

In various works, see e.g. [Com89, FW98, DPdH96, Léo95, FK06, Kra16b, CK17], it has been shown that if the initial magnetization $M_{N}(0)$ satisfies a large deviation principle with rate function $I_{0}$, then the Markov process $t \mapsto M_{N}(t)$ satisfies the large deviation principle on $]^{2} D_{\mathbb{K}}([0, \infty))$, i.e.,

$$
\mathbb{P}\left[\left(M_{N}(t)\right)_{t \geq 0} \approx \gamma\right] \approx e^{-N \mathscr{I}(\gamma)},
$$

with rate function

$$
\mathscr{I}(\gamma)= \begin{cases}I_{0}(\gamma(0))+\int_{0}^{\infty} \mathscr{L}(\gamma(s), \dot{\gamma}(s)) \mathrm{d} s & \text { if } \gamma \in \mathscr{A} \mathscr{C} \\ \infty & \text { otherwise }\end{cases}
$$

Here $\mathscr{A} \mathscr{C}$ is the space of absolutely continuous trajectories $\gamma:[0, \infty) \rightarrow \mathbb{K}$ and $\mathscr{L}:$ $\mathbb{K} \times \mathbb{R} \rightarrow[0, \infty]$ is the Lagrangian obtained by taking the Legendre transform

$$
\mathscr{L}(x, v):=\sup _{p \in \mathbb{R}}(p v-H(x, p)),
$$

of the Hamiltonian $H: \mathbb{K} \times \mathbb{R} \rightarrow \mathbb{R}$ given

(i) for the $\mathbb{R}$-space-model, by

$$
H(x, p)=\frac{1}{2} p^{2}-p W^{\prime}(x) .
$$

(ii) for the \pm 1 -space-model, by

$$
H(x, p)=v_{+}(x)\left[e^{2 p}-1\right]+v_{-}(x)\left[e^{-2 p}-1\right] .
$$

${ }^{2} D_{\mathbb{K}}([0, \infty))$ is the Skorohod space of càdlàg paths $[0, \infty) \rightarrow \mathbb{K}$, see also EK86. Section 3.5]. 
This Hamiltonian in turn is for example obtained by an operator approximation procedure introduced by Feng and Kurtz FK06. This procedure is explained informally in Redig and Wang [RW12 and rigorously for $\mathbb{R}^{d}$-valued processes in Kraaij Kra16b and Collet and Kraaij [CK17]. $H$ is derived from an operator $\mathscr{H}$ by the relation $H\left(x, f^{\prime}(x)\right)=\mathscr{H} f(x)$, where $\mathscr{H}$ satisfies $\mathscr{H} f=\lim _{N \rightarrow \infty} \mathscr{H}_{N} f$ where $\mathscr{H}_{N}$ is the operator defined by $\mathscr{H}_{N} f=N^{-1} e^{-N f} A_{N} e^{N f}$, with $A_{N}$ as defined in (9).

For any two points $a, b \in \mathbb{K}$ and time $t$, denote by

$$
S_{t}(a, b)=\inf _{\gamma \in \mathscr{A} \mathscr{C}: \gamma(0)=a, \gamma(t)=b} \int_{0}^{t} \mathscr{L}(\gamma(s), \dot{\gamma}(s)) \mathrm{d} s .
$$

$S_{t}(a, b)$ is the minimal Lagrangian action of a trajectory starting at $a$ and arriving at time $t$ at $b$.

By the contraction principle, the rate function for the large deviation principle for the magnetization $M_{N}(t)$ at time $t>0$ is given by (for $\mathscr{I}$ see (13))

$$
I_{t}(b)=\inf _{x \in \mathbb{K}}\left(I_{0}(x)+S_{t}(x, b)\right)=\inf _{\gamma \in \mathscr{A} \mathscr{C}: \gamma(t)=b} \mathscr{I}(\gamma)
$$

Definition 2.5. We call $\gamma \in \mathscr{A} \mathscr{C}$ an optimal trajectory for $S_{t}(a, b)$ (see $(16)$ ) if $\gamma(0)=a$, $\gamma(t)=b$ and $\int_{0}^{t} \mathscr{L}(\gamma(s), \dot{\gamma}(s)) \mathrm{d} s=S_{t}(a, b)$. Analogously, $\gamma$ is called an optimal trajectory for $I_{t}(b)$ (see (17)) if $\gamma(t)=b$ and $I_{t}(b)=I_{0}(\gamma(0))+\int_{0}^{\infty} \mathscr{L}(\gamma(s), \dot{\gamma}(s)) \mathrm{d} s$. Finally, $x \in \mathbb{K}$ is called a optimal starting point for $I_{t}(b)$ if $I_{t}(b)=I_{0}(x)+S_{t}(x, b)$.

In the following definition we define another way to say that (c) of the conjecture in the introduction does not hold.

Definition 2.6. We will say that $\alpha \in \mathbb{K}^{\circ}$ is a point of non-differentiability of $I_{t}$ when $I_{t}$ is not differentiable at $\alpha$.

\subsection{Preliminaries from the theory of calculus of variations}

We follow the route of studying the optimal trajectories and non-differentiabilities in the rate function by introducing techniques from the theory of calculus of variations. The first observation from classical mechanics is that optimal trajectories are known to solve the second-order Euler-Lagrange equation, which is the point of view taken in [EK10]. On equal footing, it is known that dual variables satisfy the first-order Hamilton equations.

Definition 2.7. Let $t>0$. Let $A$ be any one of the intervals $[0, t],[0, t),(0, t]$ or $(0, t)$.

Let $\gamma: A \rightarrow \mathbb{K}^{\circ}$ be absolutely continuous. If $(\gamma(s), \dot{\gamma}(s))$ is in the domain where $\mathscr{L}$ is $C^{1}$ for all $s \in A$, then the trajectory $\eta$ defined by

$$
\eta(s)=\partial_{v} \mathscr{L}(\gamma(s), \dot{\gamma}(s)),
$$

is called the dual trajectory to $\gamma$. 
Let $\gamma \in C^{1}(A, \mathbb{K})$ and $\eta \in C^{1}(A)$. We say that $(\gamma, \eta)$ satisfies the Hamilton equations, if they solve

$$
\left[\begin{array}{l}
\dot{\gamma}(s) \\
\dot{\eta}(s)
\end{array}\right]=\left[\begin{array}{c}
\partial_{p} H(\gamma(s), \eta(s)) \\
-\partial_{x} H(\gamma(s), \eta(s))
\end{array}\right] .
$$

If $(\gamma, \eta)$ satisfies the Hamilton equations and $\gamma \in C^{1}(A, \mathbb{K})$, then $\eta$ is the dual trajectory to $\gamma$ (see [CS04, Corollary A.2.7, equation (A.28)]). Moreover, there exists a $c \in \mathbb{R}$ such that $H(\gamma(s), \eta(s))=c$ for all $s \in A$.

In addition, we use the following definitions of Cannarsa and Sinestrari [CS04]. Let $d \in \mathbb{N}$ and $A \subseteq \mathbb{R}^{d}$ be open. Let $v: A \rightarrow \mathbb{R}$.

Superdifferential CS04, Definition 3.1.1] The superdifferential of $v$ at $x \in A$ is defined as $D^{+} v(x):=\left\{p \in \mathbb{R}^{d}: \lim \sup _{y \rightarrow x} \frac{v(y)-v(x)-\langle p, y-x\rangle}{|y-x|} \leq 0\right\}$. Similarly, we define a subgradient $D^{-} v(x)$. If $v$ is differentiable at $x \in A$, we write $D v(x)$ for the derivative of $v$ at $x$. Note that in that case $D^{+} v(x)=D^{-} v(x)=\{D v(x)\}$.

Reachable gradient [CS04, Definition 3.1.10] Let $v$ be locally Lipschitz. A $p \in \mathbb{R}^{d}$ is called a reachable gradient of $v$ at $x$ if there exists a sequence $\left(x_{k}\right)_{k \in \mathbb{N}}$ in $A \backslash\{x\}$ such that $v$ is differentiable at $x_{k}$ for all $k$ and $x_{k} \rightarrow x, D v\left(x_{k}\right) \rightarrow p$. We write $D^{*} v(x)$ for the set of all reachable gradients of $v$ at $x$.

Viscosity solutions CS04, Definition 5.2.1] Let $F \in C\left(A \times \mathbb{R} \times \mathbb{R}^{d}\right)$. Consider the equation

$$
F(x, v, D v)=0 .
$$

$v \in C(A)$ is called a viscosity subsolution to 20 if for all $x \in B$, we have

$$
F(x, v(x), p) \leq 0, \quad \text { for all } p \in D^{+} v(x),
$$

$v \in C(A)$ is called a viscosity supersolution to 20 if for all $x \in B$, we have

$$
F(x, v(x), p) \geq 0, \quad \text { for all } p \in D^{-} v(x) .
$$

$v$ is called a viscosity solution to 20 it it is both a sub- and a supersolution.

Local semi-concavity CS04, Definition 1.1.1 and Proposition 1.1.3] Let $B$ be a subset of $\mathbb{R}^{d}$. Let $K \subseteq B$ be compact. We say that $v$ is semi-concave on $K$ if there is some $C \geq 0$ such that

$$
\lambda v(x)+(1-\lambda) v(y)-v(\lambda x+(1-\lambda) y) \leq C \frac{\lambda(1-\lambda)}{2}|x-y|^{2}
$$

for all $x, y \in K$ such that the line from $x$ to $y$ is contained in $K$ and for all $\lambda \in[0,1]$. We call $v$ locally semi-concave on $B$ if it is semi-concave on each compact set $K \subseteq B$. Note that in CS04 these functions are called semi-concave with linear modulus, to distinguish them from a broader class of semi-concave functions. We do not need this generality here, and therefore will call "semi-concave functions with linear modulus" simply "semi-concave functions". 
Remark 2.8. With $\Phi_{2} V$ the second difference quotient of $V$ (see RS82, Section 1.2] for a definition), $\Phi_{2}$ describes the convexity and concavity properties of a function in the sense that $V$ is convex if and only if $\Phi_{2} V \geq 0$ and $V$ is concave if and only if $\Phi_{2} V \leq 0$. But it also relates to semi-concavity, as one has that if $V$ is continuous, then $V$ is semi-concave with constant $C>0$ if and only if $\Phi_{2} V \leq C$. In dHRvZ15 the $\Phi_{2} V$ is used to describe the Gibbsianity of the $\mathbb{R}$-space-model with the dynamics of independent Brownian motions, see also Remark 3.13 .

\subsection{Regularity of the rate-function}

In this section we establish the announced equivalence between the uniqueness of optimizing trajectories and differentiability of the rate function (Theorem 2.13). The main issue, which distinguishes our problems from the ones considered in [CS04, is that the maximal time of existence of solutions of the Hamilton equations, contrary to [CS04], may be finite. This causes certain divergence of the momentum at the boundary of $\mathbb{K}$. To extend the techniques to our setting, we will work under the assumptions in Assumption 4.1 .

In our setting it is natural to start with rate functions whose superdifferential is close to $-\infty$ near the left boundary and close to $\infty$ near the right boundary of $\mathbb{K}$ as this property is preserved for $I_{t}$ (see Theorem 2.11 and (22)). Moreover, we assume our initial rate function to be $C^{1}$. The space of functions that combines these two properties is called $C^{1, \partial}$ (see Definition 2.9p).

The main result (Theorem 2.13) then shows that such a rate function under the time evolution is again in $C^{1, \partial}(\mathbb{K})$ if and only if there is a unique optimizing trajectory. In Proposition 2.14 we state that the function $(t, x) \mapsto I_{t}(x)$ is the viscosity solution of the Hamilton-Jacobi equation.

Definition 2.9. For $\mathbb{K}=\mathbb{R}$ we write $\partial_{-}=-\infty$ and $\partial_{+}=\infty$, while for $\mathbb{K}=[-1,1]$ we write $\partial_{-}=-1$ and $\partial_{+}=1$.

We write $C^{k, \partial}(\mathbb{K})$ for the set of functions $g: \mathbb{K} \rightarrow \mathbb{R}$ such that $g$ is $k$ times continuously differentiable on $\mathbb{K}^{\circ}$, continuous on $\mathbb{K}$ and

$$
\lim _{x \rightarrow \partial_{+}} g^{\prime}(x)=\infty, \quad \lim _{x \rightarrow \partial_{-}} g^{\prime}(x)=-\infty .
$$

Note that for $V \in C^{1}[-1,1]$ the function $(7)$ is an element of $C^{1, \partial}[-1,1]$. Moreover, note that $I_{0} \in C^{1, \partial}(\mathbb{K})$ implies that $I_{0}$ is bounded from below and has compact sublevel sets.

Assumption 2.10. We will assume that $I_{0} \in C^{1, \partial}(\mathbb{K})$ and for $H: \mathbb{K} \times \mathbb{R} \rightarrow \mathbb{R}$ we assume

- considering the $\mathbb{R}$-space-model that (a) or (b) is satisfied:

(a) $H$ is of the form (14), where $W$ satisfies Assumption 2.4.

(b) $H$ satisfies Assumptions 4.1 and 4.11 below. 
- considering the \pm 1 -space-model that (a) or (b) is satisfied:

(a) $H$ is of the form (15), where $v_{+}, v_{-}$satisfy Assumption 2.3

(b) $H$ satisfies Assumptions 4.1 and 4.10 below.

The examples that we consider satisfy condition (a), however the proofs of the theory are based on the more general condition (b). In Appendix A we show that (a) indeed implies (b).

Theorem 2.11 (Lemma 5.1 and 5.3). Assume Assumption 2.10. Then $I_{t}$ is locally semi-concave on $\mathbb{K}^{\circ}$ for all $t \geq 0$. Moreover, $(t, x) \mapsto I_{t}(x)$ is locally semi-concave on $(0, \infty) \times \mathbb{K}^{\circ}$ and

$$
\sup _{a \in\left(0, \partial_{+}\right)} \inf _{b \geq a} \inf D^{+} I_{t}(b)=\infty, \quad \inf _{a \in\left(\partial_{-}, 0\right)} \sup _{b \leq a} \sup D^{+} I_{t}(b)=-\infty .
$$

Lemma 2.12. CSO4, Theorem 3.3.4 and 3.3.6] Let $d \in \mathbb{N}$ and $A \subseteq \mathbb{R}^{d}$ be open. Let $v: A \rightarrow \mathbb{R}$ be locally semiconcave and $x \in A$. Then $D^{+} v(x) \neq \emptyset$ and

(a) $D^{*} v(x) \subseteq \partial D^{+} v(x)$.

(b) $D^{+} v(x)=c o D^{*} v(x)$, where $c o(S)$ denotes the closed convex hull of a set $S \subseteq \mathbb{R}^{d}$.

(c) $D^{+} v(x)$ is a singleton if and only if $v$ is differentiable at $x$.

(d) If $D^{+} v(y)$ is a singleton for every $y \in A$, then $v \in C^{1}(A)$.

Theorem 2.13. Assume Assumption 2.10, Let $u:[0, \infty) \times \mathbb{K} \rightarrow[0, \infty]$ be given by

$$
u(t, x)=I_{t}(x) .
$$

Let $b \in \mathbb{K}^{\circ}, t>0$. The following are equivalent.

(a) $x \mapsto I_{0}(x)+S_{t}(x, b)$ has a unique optimizer ( $I_{t}(b)$ has a unique optimal point).

(b) $\gamma \mapsto I_{0}(\gamma(0))+\int_{0}^{\infty} \mathscr{L}(\gamma(s), \dot{\gamma}(s)) \mathrm{d} s$ has an unique optimal trajectory with $\gamma(t)=b$.

(c) $I_{t}$ is differentiable at $b$.

(d) $u$ is differentiable at $(t, b)$,

(e) $D^{*} u(t, b)$ is a singleton,

Moreover, the following are equivalent.

(A) $x \mapsto I_{0}(x)+S_{t}(x, b)$ has a unique optimizer for all $b \in \mathbb{K}^{\circ}$.

(B) $\gamma \mapsto I_{0}(\gamma(0))+\int_{0}^{t} \mathscr{L}(\gamma(s), \dot{\gamma}(s)) \mathrm{d} s$ has an unique optimal trajectory with $\gamma(t)=b$ for all $b \in \mathbb{K}^{\circ}$.

(C) $I_{t}$ is differentiable on $\mathbb{K}^{\circ}$.

(D) $I_{t} \in C^{1, \partial}(\mathbb{K})$. 
Proof. (b) $\Rightarrow$ (a). If $\gamma$ is an optimal trajectory, then $\gamma(0)$ is an optimal point for $I_{t}(b)$.

(a) $\Rightarrow(\mathrm{b})$. Suppose $x$ is a unique optimal starting point for $I_{t}(b)$, and $\gamma_{1}, \gamma_{2}$ are optimal trajectories for $I_{t}(b)$ that start in $x$. To both trajectories, we can associate dual trajectories $\eta_{1}, \eta_{2}$ so that the pairs $\left(\gamma_{1}, \eta_{1}\right)$ and $\left(\gamma_{2}, \eta_{2}\right)$ solve the Hamilton equations. Because their starting points are the same, the starting condition implies that $\eta_{i}(0)=$ $I_{0}^{\prime}(x)$. This, however, implies that the Hamilton equations are initialized with the same starting data, implying that $\gamma_{1}=\gamma_{2}$. We conclude there is a unique optimal trajectory.

By Proposition 5.2 the optimal trajectories for $I_{t}(b)$ are in one to one correspondence with the elements of $D^{*} u(t, b)$. Hence $(\mathrm{b}) \Longleftrightarrow(\mathrm{e})$.

Theorem 2.11 implies that $u$ and $I_{t}$ are locally semi-concave. By Lemma 2.12 we have $(\mathrm{d}) \Longleftrightarrow(\mathrm{e})$.

$(\mathrm{d}) \Rightarrow(\mathrm{c})$ as differentiability of $u$ at $(t, b)$ implies differentiability of $I_{t}$ at $b$.

On the other hand, if there exist two distinct optimal trajectories for $I_{t}(b)$, then the corresponding end momenta are different. By Lemma 2.12 and [CS04, Theorems 6.4.8] this implies that $D^{+} I_{t}(b)$ consists of at least two elements, i.e., $I_{t}$ is not differentiable at $b$ by Lemma 2.12 . Hence $(\mathrm{c}) \Rightarrow(\mathrm{b})$.

$(\mathrm{A}) \Longleftrightarrow(\mathrm{B}) \Longleftrightarrow(\mathrm{C})$ follow from $(\mathrm{a}) \Longleftrightarrow(\mathrm{b}) \Longleftrightarrow(\mathrm{c})$

As $I_{t}$ is locally semi-concave, $I_{t}$ is differentiable if and only if $I_{t} \in C^{1}$ on $\mathbb{K}^{\circ}$ (see Lemma 2.12. Hence (C) $\Longleftrightarrow(\mathrm{D})$ by Theorem 2.11 .

Proposition 2.14. Assume assumption 2.10. Then u (as in Theorem 2.13) is a viscosity solution to the Hamilton-Jacobi equation

$$
\partial_{t} u(t, x)+H\left(x, \partial_{x} u(t, x)\right)=0
$$

on $\mathbb{K}^{\circ} \times(0, \infty)$.

\subsection{Regularity via the push-forward of the graph of $I_{0}^{\prime}$}

Our next step is to relate optimal trajectories to the solutions of the Hamilton equations. In the following definition we present the push-forward under the Hamiltonian flow of the graph of $I_{0}^{\prime}$. In Proposition 2.16, we show that if this push-forward at time $t$ is a graph then $I_{t}$ is differentiable. Additionally, we show that overhangs in the push-forward are indications for existence of points of non-differentiability.

Definition 2.15. Assume Assumption 2.10.

(a) We write

$$
\mathscr{G}:=\left\{\left(x, I_{0}^{\prime}(x)\right): x \in \mathbb{K}^{\circ}\right\}
$$

for the graph of the derivative of $I_{0}$.

(b) For all $(x, p) \in \mathbb{K} \times \mathbb{R}$ let $\left(X_{t}^{x, p}, P_{t}^{x, p}\right)$ be the solution of the Hamilton equations (19) with initial conditions $\left(X_{0}^{x, p}, P_{0}^{x, p}\right)=(x, p)$ and up to the maximal time of existence $t_{x, p}$. (See [Per01, Theorem 2.4.1], which by Assumption 4.1(a) can also be applied in the case that $\mathbb{K}$ equals $[-1,1]$.) 
(c) For all $t>0$ we define the push-forward of $\mathscr{G}$ to be the set

$$
\mathscr{G}_{t}:=\left\{\left(X_{t}^{x, p}, P_{t}^{x, p}\right):(x, p) \in \mathscr{G}, t<t_{x, p}\right\} .
$$

(d) Fix $t>0$. We say that $\mathscr{G}_{t}$ has an overhang at $x \in \mathbb{K}^{\circ}$, if there exist $y_{1}, y_{2} \in \mathbb{R}$ with $y_{1} \neq y_{2}$ such that $\left(x, y_{1}\right),\left(x, y_{2}\right) \in \mathscr{G}_{t}$. Hence if $\mathscr{G}_{t}$ has an overhang, then it is not a graph (of a function).

Proposition 2.16. Assume Assumption 2.10. Fix $t>0$. Then

$$
\left\{(x, p): x \in \mathbb{K}^{\circ}, p \in D^{*} I_{t}(x)\right\} \subseteq \mathscr{G}_{t} .
$$

Consequently, if $\mathscr{G}_{t}$ has no overhang at $x$, then $I_{t}$ is differentiable at $x$.

On the other hand, if there are $x_{1}, x_{2} \in \mathbb{K}^{\circ}, x_{1}<x_{2}$ such that $\mathscr{G}_{t}$ has no overhang at $x_{1}$ and $x_{2}$, then

(a) $I_{t}$ is differentiable on $\left[x_{1}, x_{2}\right] \Longrightarrow \mathscr{G}_{t}$ has no overhang at $x$ for all $x \in\left(x_{1}, x_{2}\right)$.

(b) $\mathscr{G}_{t}$ has an overhang at some $x \in\left(x_{1}, x_{2}\right) \Longrightarrow I_{t}$ is not differentiable on $\left[x_{1}, x_{2}\right]$

Proof. (24) is proved in Proposition 5.2(c). If $\mathscr{G}_{t}$ has no overhang at $x$, then $D^{*} I_{t}(x)$ is a singleton and so $I_{t}$ is differentiable at $x$ by Theorem 2.13 .

We prove (a) as (b) is equivalent to (a). The interval $(a, b)$ as in Proposition 6.2 (e) is such that $\Phi_{t}(a, b)$ contains $\left(x_{i}, I_{t}^{\prime}\left(x_{i}\right)\right)$ for $i \in\{1,2\}$. As $\Phi_{t}(a, b)$ is connected, even $\left\{\left(x, I_{t}^{\prime}(x)\right): x \in\left[x_{1}, x_{2}\right]\right\} \subseteq \Phi_{t}(a, b)$. By continuity and injectivity of $\Phi_{t}$, see Proposition 6.2 (a), and the fact that $\left\{y:\left(x_{i}, y\right) \in \mathscr{G}_{t}\right\}$ are singletons for $i \in\{1,2\}$, it follows for all $x \in\left(x_{1}, x_{2}\right)$ that $\left\{y \in \mathbb{R}:(x, y) \in \mathscr{G}_{t}\right\}=\left\{y \in \mathbb{R}:(x, y) \in \Phi_{t}(a, b)\right\}$ and that this set cannot contain multiple elements.

\section{Applications of analyzing the Hamiltonian flow}

In this section we study the differentiability of $I_{t}$ by analysing the push-forward $\mathscr{G}_{t}$.

We will give a description of the results of this section in terms of the informal -but more familiar- notions high- and low-temperature. We say that a rate function $I_{0}$ is hightemperature if it is strictly convex, whereas we say that $I_{0}$ is low-temperature if it has at least two strict local minima. We say that the dynamics is high-and low-temperature if there is a high- and low-temperature $I_{0}$ such that $H\left(x, I_{0}^{\prime}(x)\right)=0$, respectively. This means that $I_{0}$ is the rate function of the stationary distributions of the dynamics with Hamiltonian $H$.

In Section 3.1 we give two general results on the preservation of differentiability, and two general results on the creation of overhangs:

Preservation at high temperature We show that for certain types of high-temperature dynamics, combined with high-temperature starting rate functions, we have differentiability of $I_{t}$ for all $t \geq 0$. 
Short-time preservation We show that 'order preserving' behaviour of the dynamics close to the boundary, combined with a starting rate-function $I_{0}$ that is strictly convex close to the boundary, implies short-time differentiability of $I_{t}$.

Large-time loss, heating We consider linearizations of the Hamiltonian flow around stationary points, which in combination with low-temperature rate-functions, create overhangs for sufficiently large times.

Large-time loss, cooling We considers areas in phase-space where the energy is negative and where the Hamiltonian flow 'rotates'. If the graph of the gradient of the rate function $I_{0}$ crosses this 'rotating' region, an overhang is created for sufficiently large times. Rotating regions occur for low-temperature Hamiltonians.

We proceed in Section 3.2 by applying these results to two sets of well known examples: Glauber dynamics for the Curie-Weiss model and interacting diffusions in a potential.

Before starting with the various applications, we introduce some notation.

Definition 3.1. For $(y, q) \in \mathbb{K}^{\circ} \times \mathbb{R}$, we write

$$
\mathbb{K}_{y, q}:=\{(x, p) \in \mathbb{K} \times \mathbb{R}: x \geq y, p \geq q\}, \quad \mathbb{Z}_{y, q}:=\{(x, p) \in \mathbb{K} \times \mathbb{R}: x \leq y, p \leq q\},
$$

and $\mathbb{K}_{y, q}^{\circ}, \mathbb{7}_{y, q}^{\circ}$ for the interiors of $\mathbb{K}_{y, q}, \mathbb{7}_{y, q}$ in $\mathbb{K} \times \mathbb{R}$.

E.g., for $\mathbb{K}=[-1,1], \mathbb{K}_{y, q}=[y, 1] \times[q, \infty)$ and $\mathbb{K}_{y, q}^{\circ}=(y, 1] \times(q, \infty)$.

Definition 3.2. Assume Assumption 2.10.

(a) Let $A \subseteq \mathbb{K} \times \mathbb{R}$. We say that $H$ preserves $A$, or $A$ is preserved under $H$, if all trajectories $(\gamma, \eta)$ satisfying the Hamilton equations with $(\gamma(0), \eta(0)) \in A$ stay in $A$ during their life-time, i.e., $(\gamma(s), \eta(s)) \in A$ for all $s<t_{\gamma(0), \eta(0)}$ if $(\gamma(0), \eta(0)) \in A$.

(b) If $\left(x_{0}, p_{0}\right) \in \mathbb{K} \times \mathbb{R}$ is such that $H$ preserves the set $\left\{\left(x_{0}, p_{0}\right)\right\}$, then we call $\left(x_{0}, p_{0}\right)$ stationary under $H$. Mostly we consider stationary points of the form $\left(x_{0}, 0\right)$, in such case we will also say that $x_{0}$ is stationary.

(c) We say that $H: \mathbb{K} \times \mathbb{R} \rightarrow \mathbb{R}$ preserves order on a subset $A \subseteq \mathbb{K} \times \mathbb{R}$ if $A$ is preserved under $H$ and if for $\left(x_{1}, p_{1}\right),\left(x_{2}, p_{2}\right) \in \mathbb{K} \times \mathbb{R}$ and $t<t_{x_{1}, p_{1}} \wedge t_{x_{2}, p_{2}}$,

$$
x_{1}<x_{2}, p_{1}<p_{2} \quad \Longrightarrow \quad X_{t}^{x_{1}, p_{1}}<X_{t}^{x_{2}, p_{2}}, P_{t}^{x_{1}, p_{1}}<P_{t}^{x_{2}, p_{2}} .
$$

(d) We say that $H$ preserves order at infinity if there are $\left(y_{-}, q_{-}\right),\left(y_{+}, q_{+}\right) \in \mathbb{K}^{\circ} \times \mathbb{R}$ such that $H$ preserves order on $\mathbb{7}_{y_{-}, q_{-}}$and $\mathbb{K}_{y_{+}, q_{+}}$.

(e) We say that $I_{0}$ is strictly convex at infinity if there is some compact set $K \subseteq \mathbb{K}^{\circ}$ such that $I_{0}^{\prime}$ is strictly increasing on the complement of $K$.

Two effective methods to determine that $\mathscr{G}_{t}$ has overhangs are via rotating areas in the Hamiltonian flow and via linearizations of the flow, of which the definition follows. 
Definition 3.3. Assume Assumption 2.10 Let $x_{0} \in \mathbb{K}^{\circ}$ be a stationary point for the Hamiltonian flow, i.e., $\partial_{p} H\left(x_{0}, 0\right)=0$. We say that the Hamiltonian flow admits a $C^{1}$ linearization at $\left(x_{0}, 0\right)$ if there exist open neighbourhoods $U, V \subseteq \mathbb{R}^{2}$ of $\left(x_{0}, 0\right)$, and a $C^{1}$-diffeomorphism $\Psi: U \rightarrow V$ such that $\Psi\left(x_{0}, 0\right)=\left(x_{0}, 0\right)$ and $D \Psi\left(x_{0}, 0\right)=\mathbb{1}$ (the identity matrix) and such that a trajectory $(\gamma, \eta)$ with values in $U$ solves the Hamilton equations 19$)$ if and only if $(\xi, \zeta)=\Psi(\gamma, \eta)$ solves

$$
\left[\begin{array}{c}
\dot{\xi}(s) \\
\dot{\zeta}(s)
\end{array}\right]=\nabla^{2} H\left(x_{0}, 0\right)\left[\begin{array}{c}
\xi(s)-x_{0} \\
\zeta(s)
\end{array}\right],
$$

where $\nabla^{2} H\left(x_{0}, 0\right)$ denotes the Hessian of $H$ at $\left(x_{0}, 0\right)$, so that with

$$
m:=\partial_{x} \partial_{p} H\left(x_{0}, 0\right), \quad c:=\partial_{p}^{2} H\left(x_{0}, 0\right),
$$

it has the form

$$
\nabla^{2} H\left(x_{0}, 0\right)=\left[\begin{array}{cc}
\partial_{x} \partial_{p} H\left(x_{0}, 0\right) & \partial_{p}^{2} H\left(x_{0}, 0\right) \\
-\partial_{x}^{2} H\left(x_{0}, 0\right) & -\partial_{x} \partial_{p} H\left(x_{0}, 0\right)
\end{array}\right]=\left[\begin{array}{cc}
m & c \\
0 & -m
\end{array}\right],
$$

note that $-\partial_{x}^{2} H\left(x_{0}, 0\right)=0$ because $H(x, 0)=0$ for all $x$ and that $c>0$.

Remark 3.4. Note that we include in the definition that $D \Psi\left(x_{0}, 0\right)=\mathbb{1}$ which is not a standard assumption. However, we need this assumption to connect the dynamics of the graph $\mathscr{G}$ under the Hamiltonian flow, to that of the dynamics of the tangent of the push-forward of $\mathscr{G}$.

\subsection{Preservation of differentiability and the creation of overhangs}

The following theorem relates preservation of order under $H$ and strict convexity of $I_{0}$ to differentiability of $I_{t}$. The proof can be found in Section 7 .

Theorem 3.5. Assume Assumption 2.10.

(a) Suppose that $I_{0}$ is strictly convex and that $\mathscr{G}$ is contained in a set on which $H$ preserves order. Then $I_{t} \in C^{1, \partial}(\mathbb{K})$ for all $t \geq 0$.

(b) Assume that $I_{0}$ is $C^{2}$ on $\mathbb{K}^{\circ}$, strictly convex at infinity and that $H$ preserves order at infinity. Then there is a $t_{0}>0$ such that $I_{t} \in C^{1, \partial}(\mathbb{K})$ for all $0 \leq t \leq t_{0}$.

The next theorem gives conditions under which overhangs are created. The proofs of (a) and (b) can be found in Section 8 .

Theorem 3.6. Assume Assumption 2.10.

(a) Suppose $x_{0} \in \mathbb{K}^{\circ}$ is such that $I_{0}^{\prime}\left(x_{0}\right)=0$ and that $\left(x_{0}, 0\right)$ is a stationary point. Let $m, c$ be as in (26). Suppose $I_{0}$ is $C^{2}$ in a neighbourhood of $x_{0}$ with $I_{0}^{\prime \prime}\left(x_{0}\right)<$ 
$\min \left\{-\frac{2 m}{c}, 0\right\}$. In addition, assume that the Hamiltonian flow admits a $C^{1}$ linearization at $\left(x_{0}, 0\right)$ (a sufficient condition for this is that $H$ is $C^{\infty}$ and $m \neq 0^{3}$ ). Let

$$
t_{0}:= \begin{cases}-\frac{1}{2 m} \log \left(1+\left(\frac{c}{2 m} I_{0}^{\prime \prime}\left(x_{0}\right)\right)^{-1}\right) & \text { if } m \neq 0, \\ -\frac{1}{c I_{0}^{\prime \prime}\left(x_{0}\right)} & \text { if } m=0 .\end{cases}
$$

Then $\mathscr{G}_{t}$ contains an overhang at $x_{0}$ for all $t>t_{0}$.

(b) Suppose that $H$ is $C^{3}$. In addition suppose that $m_{1}, m_{2} \in \mathbb{K}^{\circ}$ are two points such that $m_{1}<m_{2}$ and

(i) $\partial_{p} H\left(m_{1}, 0\right)=0=\partial_{p} H\left(m_{2}, 0\right)$ and $\partial_{p} H(x, 0) \neq 0$ for all $x \in\left(m_{1}, m_{2}\right)$,

(ii) $\partial_{x} \partial_{p} H\left(m_{1}, 0\right) \neq 0$ and $\partial_{x} \partial_{p} H\left(m_{2}, 0\right) \neq 0$.

Suppose that $\mathscr{G} \cap\left\{(x, p) \in\left(m_{1}, m_{2}\right) \times \mathbb{R}: H(x, p)<0\right\} \neq \emptyset$. Then there is a time $t_{0}>0$ such that there is a $x_{0} \in\left(m_{1}, m_{2}\right)$ such that $\mathscr{G}_{t}$ contains an overhang at $x_{0}$ for all $t \geq t_{0}$.

Remark 3.7. In (a) $t_{0}$ is the time that the line $x \mapsto x\left(1, I_{0}^{\prime \prime}\left(x_{0}\right)\right)$ is transformed into a vertical line under the linearized flow.

Regarding (b), in Section 8 we show that if a set $A$ satisfies certain properties, then the Hamiltonian flow rotates over the boundary $\partial A$ of $A$, which means that every Hamilton path started on $\partial A$ will move along $\partial A$ and return to its initial point in finite time.

We prove that the conditions of (b) imply the existence of such a set in Lemma 8.6 .

\subsection{Explicit results for two main classes of examples}

In the present section, we analyze particular canonical instances of the \pm 1 -space-model and the $\mathbb{R}$-space-model:

(i) for the $\mathbb{R}$-space-model we consider $H$ and $I$ of the form

$$
H(x, p)=\frac{1}{2} p^{2}-p W_{b}^{\prime}(x), \quad I_{0}(x)=W_{a}(x),
$$

for $a, b \in \mathbb{R}$, where $W_{d}(x)=\frac{1}{4} x^{4}-\frac{1}{2} d x^{2}$ for $d \in \mathbb{R}$.

(ii) for the \pm 1 -space-model we consider $H$ and $I$ of the form

$$
\begin{aligned}
H(x, p) & =\frac{1-x}{2} e^{\beta x+h}\left[e^{2 p}-1\right]+\frac{1+x}{2} e^{-\beta x-h}\left[e^{-2 p}-1\right], \\
I_{0}(x) & =\frac{1-x}{2} \log (1-x)+\frac{1+x}{2} \log (1+x)-\frac{1}{2} \alpha x^{2}-\theta x+C,
\end{aligned}
$$

where $\alpha, \beta \geq 0, \theta, h \in \mathbb{R}$ and $C \in \mathbb{R}$ is such that $\inf _{x \in[-1,1]} I_{0}(x)=0$. I.e., we consider Curie-Weiss dynamics with inverse temperature $\beta$ and a starting rate function that corresponds to an inverse temperature $\alpha$.

\footnotetext{
${ }^{3}$ See Theorem 8.1
} 
In Proposition 3.9 we consider conditions sufficient for $H$ to preserve order. In Theorem 3.10 we apply the results of Theorems 3.5 and 3.6 for various choices of $a$ and $b$ or of $\alpha, \beta, h$ and $\theta$. In Theorem 3.11, we show that for specific choices of $\alpha$ and $\theta$ one obtains recovery of differentiability. We restrict our analysis mostly to $\beta=h=0$, i.e., infinite-temperature dynamics and zero dynamical external magnetic field. For other parameters we expect that one can obtain similar results at the expense of more involved computations, which go beyond the scope of this text.

In the proof of Theorem 3.10 and Theorem 3.11, we use the creation of overhangs proved in Theorem 3.6 to show non-differentiability with the help of Proposition 2.16.

Remark 3.8. One can rewrite $H$ as in $(29)$ and compute the following derivatives, which will be used later in proofs. We spare the reader the computations.

$$
\begin{aligned}
H(x, p) & =2 \sinh (p)[\sinh (\beta x+h+p)-x \cosh (\beta x+h+p)], \\
\partial_{p} H(x, p) & =2 \sinh (\beta x+h+2 p)-2 x \cosh (\beta x+h+2 p) \\
\partial_{x} H(x, p) & =-2 \sinh (p)[(1-\beta) \cosh (\beta x+h+p)+\beta x \sinh (\beta x+h+p)], \\
\partial_{p} \partial_{x} H(x, p) & =2(\beta-1) \cosh (\beta x+h+2 p)-2 \beta x \sinh (\beta x+h+2 p), \\
\partial_{x}^{2} H(x, p) & =-2 \beta \sinh (p)[(2-\beta) \sinh (\beta x+h+p)+\beta x \cosh (\beta x+h+p)], \\
\partial_{p}^{2} H(x, p) & =4 \cosh (\beta x+h+2 p)-4 x \sinh (\beta x+h+2 p) .
\end{aligned}
$$

Note that $H(x, p)=0$ if and only if either $p=0$ or $h+p=\operatorname{arctanh}(x)-\beta x$ and that $\partial_{p} H(x, p)=0$ if and only if $2 p=\operatorname{arctanh}(x)-\beta x-h$.

For $I_{0}$ as in (30) we have

$$
\begin{aligned}
& I_{0}^{\prime}(x)=\operatorname{arctanh}(x)-\alpha x-\theta, \\
& I_{0}^{\prime \prime}(x)=\frac{1}{1-x^{2}}-\alpha .
\end{aligned}
$$

Proposition 3.9. (a) Assume Assumption 2.3 for the \pm 1 -space-model, with $H$ as in (15). In addition, suppose $v_{+}^{\prime \prime}(1)<0$ and $v_{-}^{\prime \prime}(-1)<0$ on a neighbourhood of -1 . Then $H$ preserves order at infinity.

Let $H$ as in $(29)$. Then $H$ preserves order at infinity for all $\beta \geq 0$ and $h \in \mathbb{R} ;$ for $h=0$ and $\beta \in[0,2], H$ preserves order on $\mathbb{\lambda}_{0,0}^{\circ} \cup\{0\} \cup \mathfrak{K}_{0,0}^{\circ}$; and, for $\beta=h=0$, $H$ preserves order on the entire space $[-1,1] \times \mathbb{R}$.

(b) Assume Assumption 2.4 for the $\mathbb{R}$-space-model, with $H$ as in (14). Let $y, z \in \mathbb{R}$. If $W^{\prime \prime \prime}(x) \leq 0$ for all $x \leq y$, then $H$ preserves order on $\mathbb{7}_{y, 0}^{\circ}$. If $W^{\prime \prime \prime}(x) \geq 0$ for all $x \geq z$, then $H$ preserves on $\mathbb{K}_{z, 0}^{\circ}$.

If such $y$ and $z$ as above exist, then $H$ preserves order at infinity. Such $y$ and $z$ exist, e.g., for $W$ of the form $W(x)=\sum_{i=1}^{2 k} a_{i} x^{i}$ with $a_{2 k}>0$ and thus for $W$ as in $(28)$. 


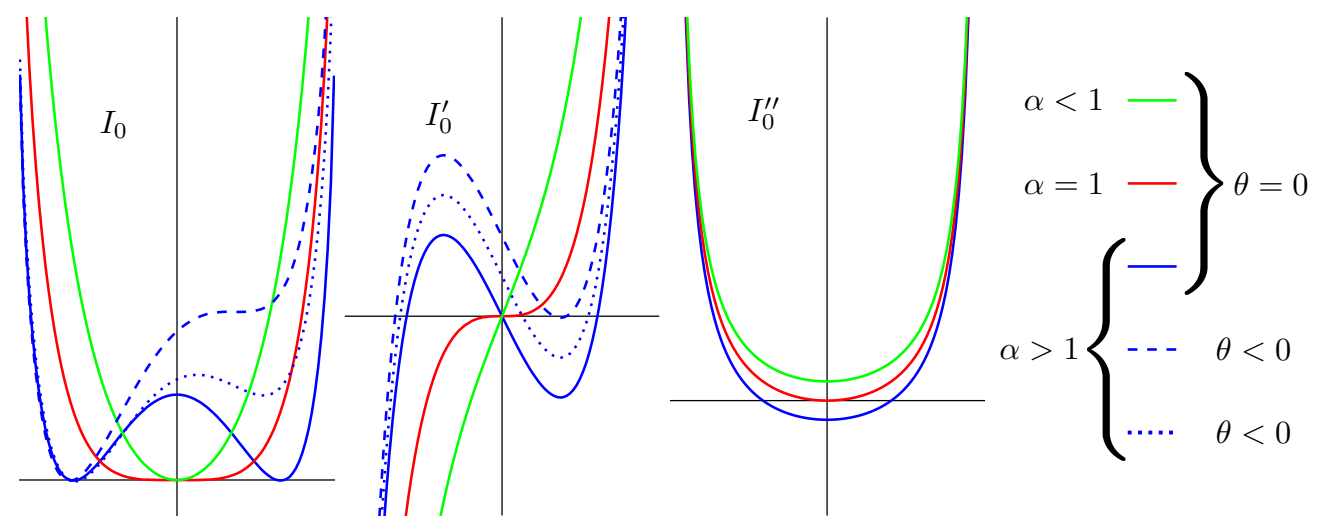

Figure 1: $I_{0}$ of (30), $I_{0}^{\prime}$ and $I_{0}^{\prime \prime}$ for different values of $\alpha$ and $\theta$.

Proof. (a) By Proposition 7.2 and because Assumption 4.1(e) holds (see Lemma A.1), it is sufficient to show that $\partial_{x}^{2} H<0$ on quadrants $\mathbb{K}_{x, p}$ and $\mathbb{\lambda}_{x, p}$ for $x, p$ close to $(1, \infty)$ and $(-1,-\infty)$, respectively. Those $x, p$ can be found as $\partial_{x}^{2} H(x, p)=v_{+}^{\prime \prime}(x)\left[e^{2 p}-1\right]+$ $v_{-}^{\prime \prime}(x)\left[e^{-2 p}-1\right]$ and $v^{\prime \prime}$ is continuous.

For $H$ as in (29), i.e., $H$ as in (15) with $v_{-}$and $v_{+}$as in (11) we have $v_{+}^{\prime \prime}(1)=-\beta e^{\beta+h}$ and $v_{-}^{\prime \prime}(-1)=-\beta e^{\beta-h}$ which are both $<0$ for all $\beta>0$ and $h \in \mathbb{R}$.

For $\beta=0$ and $h \in \mathbb{R}$ it follows that $(x, p) \mapsto \partial_{x} H(x, p)$ is a decreasing function at infinity, whence $H$ preserves at infinity by Proposition 7.2 .

For $\beta \in(0,2]$ and $h=0$, by (35) we see that $\partial_{x}^{2} H(x, p)<0$ on $7_{0,0}^{\circ} \cup K_{0,0}^{\circ}$. As $\partial_{p} H(0, p)=2 \sinh (2 p)$, and $H(x, p)=0$ if and only if either $p=0$ or $p=\operatorname{arctanh}(x)-\beta x$, a Hamilton trajectory in $7_{0,0}^{\circ}$ could only leave this set via the point $(x, 0)$ with $H(x, 0)=$ 0 , which is a stable point (see Remark 3.8) and therefore cannot be reached. Hence $H$ preserves $7_{0,0}^{\circ}$ and similarly $\mathfrak{K}_{0,0}^{\circ}$. Proposition 7.2 implies that $H$ preserves order on both $7_{0,0}^{\circ}$ and $\mathbb{K}_{0,0}^{\circ}$, and therefore on the union of those sets with $\{0\}$.

For $\beta=h=0$, by (33) $\partial_{x} H(x, p)=-2 \sinh (p) \cosh (p)$ which is decreasing as a function of $(x, p)$, so that $H$ preserves order on the whole space $[-1,1] \times \mathbb{R}$.

(b) Note that $\partial_{x}^{2} H(x, p)=-p W^{\prime \prime \prime}(x)$, which immediately establishes the claim.

In addition to applying the abstract results of Section 3.1, we use Proposition 2.16 to show that in particular settings overhangs do induce non-differentiabilities of the rate function. The proofs of the following results are given in Section 9 .

Theorem 3.10. For the $\mathbb{R}$-space-model and the \pm 1 -space-model, under the above assumptions, we have the following scenarios for the following $a, b$ and $\alpha, \beta$ :

(a) $[a=b],[\alpha=\beta, \theta=h]$, equilibrium. We have $I_{0}=I_{t}$ for all $t \geq 0$.

(b) $[a, b \in \mathbb{R}],[\alpha, \beta \geq 0, \theta, h \in \mathbb{R}]$, short-time differentiability.

There is a $t_{0}>0$ such that for $t \leq t_{0}$ we have $I_{t} \in C^{1, \partial}(\mathbb{K})$. 
(c) $[a \leq 0],[\alpha \leq 1,0<\beta \leq 2, \theta \in \mathbb{R}, h=0]$, high-temperature starting profile. For all $t \geq 0$ we have $I_{t} \in C^{1, \partial}(\mathbb{K})$.

(d) $[a>b \vee 0, b \neq 0],[\alpha>\beta \vee 1, \beta \neq 1, \theta=h=0]$, heating up a low-temperature starting profile. There is an overhang at $x=0$ for all $t \geq t_{1}$ where

(i) for the $\mathbb{R}$-space-model $t_{1}=-\frac{1}{b} \log \left(\frac{a-b}{a}\right)$.

(ii) for the \pm 1 -space-model $t_{1}=-\frac{1}{4(1-\beta)} \log \left(\frac{\beta-\alpha}{1-\alpha}\right)$.

(e) $[0<a<b],[1<\alpha<\beta, \theta=h=0]$, cooling down a low-temperature starting profile. There is some $t_{2}$ such that for all $t \geq t_{2}$ there exist at least two points $x_{-}, x_{+}$of non-differentiability of $I_{t}$ with $m_{-}<x_{-}<0<x_{+}<m_{+}$, where

(i) for the $\mathbb{R}$-space-model $m_{ \pm}= \pm \sqrt{b}$.

(ii) for the \pm 1 -space-model $m_{ \pm}$are the solutions to $\operatorname{arctanh}(x)=\beta x \mathbb{H}^{4}$

Theorem 3.11 (Loss and recovery). Let $\mathbb{K}=[-1,1]$ and $H$ and $I_{0}$ be as in (29) and (30) with $\beta=h=0$.

(a) For $\alpha \geq 0$ and $\theta \in \mathbb{R}$ such that $I_{0}^{\prime}=0$ has a unique solution, there is some time $t^{*}$ such that $I_{t} \in C^{1, \partial}[-1,1]$ for $t \geq t^{*}$.

(b) For all $\alpha>1$ there exists a $\kappa>0$ such that for all $\theta \in \mathbb{R}$ with $|\theta|>\kappa$, there are times $t_{0}<t_{1} \leq t_{2}$ such that

(i) $I_{t} \in C^{1, \partial}[-1,1]$ for $t<t_{0}$,

(ii) $\mathscr{G}_{t}$ contains an overhang for $t \in\left(t_{0}, t_{2}\right)$ and $I_{t}$ is non-differentiable for $t \in$ $\left(t_{0}, t_{1}\right)$,

(iii) $I_{t} \in C^{1, \partial}[-1,1]$ for $t>t_{2}$.

\subsection{Remarks on the results and comparison with the literature}

Remark 3.12. Our method to verify non-differentiability for Theorem $3.10[$ (d) is based on Proposition 2.16 up to the time at which the push-forward of $\mathscr{G}$ falls apart in three separate curves, cf. Proposition 6.2.

Similarly, in our proof of Theorem 3.11|(b), we actually have $t_{1}<t_{2}$ (see also Remark 9.1], i.e., for $t \in\left[t_{1}, t_{2}\right)$ there is an overhang but again Proposition 2.16 cannot be used to conclude that $I_{t}$ is non-differentiable.

Remark 3.13. In dHRvZ15 the $\mathbb{R}$-space-model with $H(x, p)=\frac{1}{2} p^{2}$ and $I_{0}(x)=\frac{1}{2} x^{2}+$ $V(x)$, where $V \in C^{1}(\mathbb{R},[0, \infty))$ is considered. We show that the existence of an overhang as in Theorem 3.6(a) agrees with the non-differentiability claimed in dHRvZ15. Note that the Hamiltonian flow admits a $C^{1}$ linearization by the identity map as the flow itself is linear. In dHRvZ15, Corollary 1.12] it is shown that $I_{t}(b)$ has a unique global minimiser for all $b \in \mathbb{R}$ if and only if $\Phi_{2} V>-\frac{1+t}{2 t}$. Hence $I_{t}$ is not differentiable if

\footnotetext{
${ }^{4}$ As $\beta>1$ such $m_{-}$and $m_{+}$exist.
} 
$\Phi_{2} V \ngtr-\frac{1+t}{2 t}$, which by dHRvZ15, Lemma 5.9] is the case when there exists an $x_{0}$ for which $V^{\prime \prime}\left(x_{0}\right)+\frac{1}{2}<-\frac{1}{t}$, which is the same as $t>t_{0}$ for $t_{0}$ as in (27).

Theorem 3.5 also agrees with dHRvZ15, Corollary 1.12] in case $I_{0}(x)=\frac{1}{2} x^{2}+V(x)$ is strictly convex (at infinity). However, the setting in dHRvZ15 allows $I_{0}$ not to be in $C^{1, \partial}$. This is the case for, e.g., $V(x)=1+\cos \left(x^{2}\right)$ as in [dHRvZ15, Example 1.16]. Here one has immediate loss of uniqueness of minimisers and therefore an immediate loss of differentiability, which can be proved by [CS04, Corollary 6.4.4 and Theorem 6.4.8] in the same way as in the proof of Theorem 2.13 .

Remark 3.14. Consider a stationary point $x_{0}$. The condition that $\partial_{p} \partial_{x} H\left(x_{0}, 0\right) \neq 0$ in both Theorem 3.6 (a) and (b) is reflected by the exclusion of having $b=0$ and $\beta=1$, i.e., $\partial_{p} \partial_{x} H(0,0)=0$, in Theorem 3.10 (d).

In the literature on dynamical systems, the failure of $\partial_{p} \partial_{x} H\left(x_{0}, 0\right) \neq 0$ implies that $\left(x_{0}, 0\right)$ is a non-hyperbolic fixed point of the Hamiltonian flow. This can be considered to be critical behaviour: for a non-hyperbolic fixed point, the first-order approximation does not describe the global behaviour of the flow around this point.

This is similar to the statement that $\alpha=1$ is critical for the Curie-Weiss model: the first-order approximation $I_{0}^{\prime}(0)$, with $I_{0}$ as in $(30)$, of the rate function $I_{0}$ at the point 0 vanishes for $\alpha=0$, indicating a transition from a convex to a non-convex rate-function.

Remark 3.15. Both the idea of linearization and rotation already appeared for the Lagrangian flow in EK10. The idea of considering the Hamiltonian flow instead of the Lagrangian flow already appeared in [Kra16c, Chapter 5]. As our methods do not depend on a specific model, we recover part of the results of [EK10], however some of our results are slightly weaker:

Using explicit calculations, EK10, Theorem 1.3] obtains the result in Theorem 3.10||(c) for all $\beta$ instead of $\beta \leq 2$.

Our result in (d) for $1 \vee \beta<\alpha$ is sub-optimal. [KLN07, EK10 show that points of non-differentiability occur before the linearized system assures that we have a point of non-differentiability at 0 . In this setting, there is a different mode, apart from the rotation around 0 that creates the overhang. This mode can easily be identified by using pictorial analysis based on the Hamiltonian flow.

\section{A study of the Hamiltonian dynamics, optimizers, and the time-evolved rate function}

The extension of calculus of variations to a setting where the Hamiltonian trajectories may have finite maximal times of existence needs a treatment of the behaviour of the Hamiltonian flow close to the boundary. This analysis will be carried out under general conditions that are introduced in Sections 4.1 and 4.2. We will show that these conditions imply that Hamiltonian trajectories are pushed away from the boundary, and can only arrive at the boundary with infinite momentum at their maximal time of existence. 
In Section 4.3, we show that optimizers of $I_{t}(b)$ together with their dual trajectories, are solutions of the Hamilton equations. In Section 5 we establish the regularity properties of the rate function that we introduced in Sections 2.6 and 2.7 .

\subsection{Conditions on Hamiltonian and properties of the Hamiltonian flow}

Below, we introduce the main assumptions of our paper. The assumptions (a)-(e) fall apart in two natural groups. (a) and (b) are there to study the Hamiltonian flow in open subsets of $\mathbb{K} \times \mathbb{R}$. In particular, these conditions suffice to apply the methods described in [CS04], compare with Conditions (L1)-(L4) on $\mathscr{L}$ in [CS04], as long as Hamiltonian trajectories remain inside such open sets.

The assumptions (c)-(e) are made to study behaviour of Hamiltonian trajectories at the boundary, or to show that Hamiltonian trajectories stay away from the boundary.

Assumption 4.1. The Hamiltonian $H: \mathbb{K} \times \mathbb{R} \rightarrow \mathbb{R}$ satisfies $H(x, 0)=0$ for all $x$ and

(a) $H$ is $C^{2}$ and $\partial_{p}^{2} H(x, p)>0$ for all $(x, p) \in \mathbb{K} \times \mathbb{R}$.

If $\mathbb{K}=[-1,1]$, then we additionally assume that there exist an $\epsilon>0$ and a twice continuously differentiable function $\tilde{H}:(-1-\epsilon, 1+\epsilon) \times \mathbb{R} \rightarrow \mathbb{R}$ such that $H$ equals $\tilde{H}$ on $[-1,1] \times \mathbb{R}$.

(b) For every compact set $K \subseteq \mathbb{K}^{\circ}$, there exists a function $\theta_{K}:[0, \infty) \rightarrow[0, \infty)$, with the properties

(i) $\lim _{r \rightarrow \infty} r^{-1} \theta_{K}(r)=\infty$,

(ii) for every $M \geq 0$, there is a $k_{M} \geq 0$ such that $\theta_{K}(r+m) \leq k_{M}\left(1+\theta_{K}(r)\right)$ for all $m \in[0, M]$ and $r \geq 0$,

(iii) there exists $c_{K}$ such that $\mathscr{L}(x, v) \geq \theta_{K}(|v|)-c_{K}$ for all $x \in K$ and $v \in \mathbb{R}$,

(iv) there exists $C_{K}$ such that $\left|\partial_{x} \mathscr{L}(x, v)\right|+\left|\partial_{v} \mathscr{L}(x, v)\right| \leq C_{K} \theta_{K}(|v|)$ for all $x \in K$ and $v \in \mathbb{R}$.

(c) For each compact $K \subseteq \mathbb{K}^{\circ}$, we have $\lim _{|p| \rightarrow \infty} \inf _{x \in K} \frac{H(x, p)}{|p|}=\infty$.

If $\mathbb{K}=[-1,1]$, then we additionally assume

$$
\lim _{p \rightarrow \infty} \frac{H(-1, p)}{p}=\infty, \quad \lim _{p \rightarrow-\infty} \frac{H(1, p)}{-p}=\infty .
$$

(d) We have

$$
\lim _{x \rightarrow \partial_{-}} \underset{p \in \mathbb{R}}{\operatorname{argmin}} H(x, p)=-\infty, \quad \lim _{x \rightarrow \partial_{+}} \underset{p \in \mathbb{R}}{\operatorname{argmin}} H(x, p)=\infty
$$

(e) There exists a sequence $\left(\left(y_{n}^{+}, q_{n}^{+}\right)\right)_{n \in \mathbb{N}}$ in $\mathbb{K}^{\circ} \times(0, \infty)$ with $\lim _{n \rightarrow \infty}\left(y_{n}^{+}, q_{n}^{+}\right)=$ $\left(\partial_{+}, \infty\right)$ and

$$
\begin{aligned}
\partial_{p} H\left(y_{n}^{+}, q\right) & \geq 0 \text { for } q \geq q_{n}^{+}, \\
-\partial_{x} H\left(y, q_{n}^{+}\right) & \geq 0 \text { for } y \geq y_{n}^{+},
\end{aligned}
$$


and there exists a sequence $\left(\left(y_{n}^{-}, q_{n}^{-}\right)\right)_{n \in \mathbb{N}}$ in $\mathbb{K}^{\circ} \times(-\infty, 0)$ with $\lim _{n \rightarrow \infty}\left(y_{n}^{-}, q_{n}^{-}\right)=\left(\partial_{-},-\infty\right)$ and

$$
\begin{aligned}
\partial_{p} H\left(y_{n}^{-}, q\right) & \leq 0 \text { for } q \leq q_{n}^{-}, \\
-\partial_{x} H\left(y, q_{n}^{-}\right) & \leq 0 \text { for } y \leq y_{n}^{-},
\end{aligned}
$$

Remark 4.2. - By Assumption $4.1(\mathrm{e}) \bigsqcup_{y_{n}^{+}, q_{n}^{+}}$and $\prod_{y_{n}^{-}, q_{n}^{-}}$are preserved under $H$.

- Assumption 4.1|(c) implies that for every compact set $K \subseteq \mathbb{K}^{\circ}$ and $c \in \mathbb{R}$ the set $(K \times \mathbb{R}) \cap H^{-1}(-\infty, c]$ is compact.

Lemma 4.3. Let $\mathbb{K}=[-1,1]$. Then Assumption 4.1(a) and (d) imply

$$
\begin{array}{cc}
v_{-}:=\lim _{p \rightarrow-\infty} \partial_{p} H(-1, p) \geq 0, & v_{+}:=\lim _{p \rightarrow \infty} \partial_{p} H(1, p) \leq 0, \\
\lim _{p \rightarrow-\infty} \frac{H(-1, p)}{p}=v_{-} \geq 0, & \lim _{p \rightarrow \infty} \frac{H(1, p)}{p}=v_{+} \leq 0,
\end{array}
$$

and that the Hamiltonian vector field on the boundary points inwards:

$$
\partial_{p} H(-1, p)>0, \quad \partial_{p} H(1, p)<0 .
$$

Moreover, these assumptions together with Assumption 4.1(n) imply that for all $c \in \mathbb{R}$, for every $(a, q) \in(-1,1) \times(0, \infty)$ and $(b, r) \in(-1,1) \times(-\infty, 0)$ the set (see Figure $4.1(a)$ )

$$
\left(\mathfrak{K}_{a, q}^{\circ} \cup \mathbb{T}_{b, r}^{\circ}\right)^{c} \cap H^{-1}(-\infty, c]
$$

is compact.

Proof. Because (a) we have that $p \mapsto H(x, p)$ is strictly convex and thus that $q=$ $\operatorname{argmin}_{p \in \mathbb{R}} H(x, p)$ if and only if $\partial_{p} H(x, p)=0$. Moreover, if $q>\operatorname{argmin}_{p \in \mathbb{R}} H(x, p)$ then $\partial_{p} H(x, p)>0$. Therefore (d) implies that $\partial_{p} H(-1, q)=\lim _{x \rightarrow-1} \partial_{p} H(x, p) \geq 0$. This in turn implies 43 . By the strict convexity of $p \mapsto H(x, p)$ we have

$$
q<p \quad \Longrightarrow \quad \partial_{p} H(x, q)<\frac{H(x, q)-H(x, p)}{q-p}<\partial_{p} H(x, p) .
$$

Hence (43) implies (44) and 45).

Note that $\left(K_{a, q}^{\circ} \cup \mathbb{7}_{b, r}^{\circ}\right)^{c}$ is a subset of the union of the compact set $[-1,1] \times[r, q]$ with $[-1, u] \times[0, \infty),[v, 1] \times(-\infty, 0]$ and $[u, v] \times \mathbb{R}$ for any $u<a$ and $v>b$. Hence to show that (46) is compact, it is by Remark 4.2 sufficient to show that there exist $u$ and $v$ in $(-1,1)$ such that $([-1, u] \times[0, \infty)) \cap H^{-1}(-\infty, c]$ and $([v, 1] \times(-\infty, 0]) \cap H^{-1}(-\infty, c]$ are compact.

By (d) there exists an $u$ such that $\operatorname{argmin}_{p \in \mathbb{R}} H(x, p)<0$ for all $x \leq u$. Therefore there exists an $\epsilon>0$ such that $\partial_{p} H(x, p)>\epsilon$ for all $x \in[-1, u]$ and all $p \geq 0$ (see also (47)). As $H(x, 0)=0$ for all $x$, this implies that there exists an $M>0$ such that for $p \geq$ $M$ and $x \in[-1, u]$ we have $H(x, p)>c$. This proves that $([-1, u] \times[0, \infty)) \cap H^{-1}(-\infty, c]$ is compact. The existence of a $v$ can be proved in the same way. 
We proceed with exploring the behaviour of solutions to the Hamilton equations 'close' to the boundary. This analysis will crucially depend on Assumptions 4.1 (c), (d) and (e). We start with a result that captures the idea that the Hamiltonian flow points away from the 'boundary' points $\partial_{-}$and $\partial_{+}$, unless one starts with very low and very high momentum, respectively (see also Figure 4.1(b)).

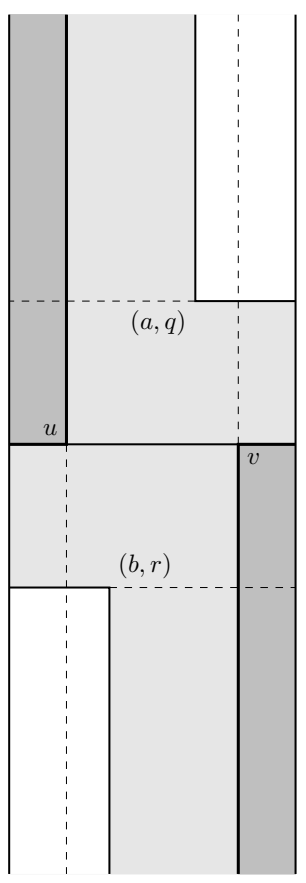

(a)

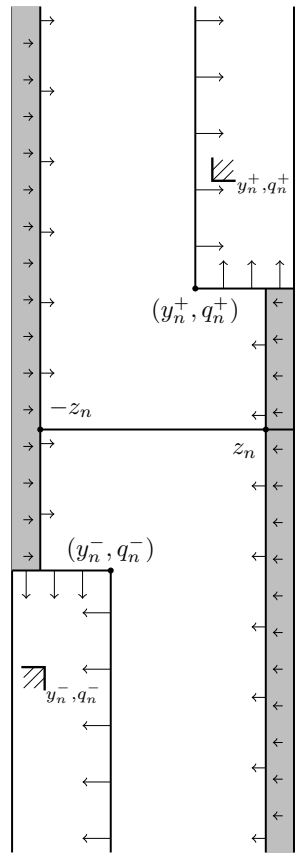

(b)

Figure 2: (a) complement of quadrants, (b) push from boundary.

Lemma 4.4. Let $H$ satisfy Assumption 4.1. There exists a sequence $\left(z_{n}\right)_{n \in \mathbb{N}}$ in $\left(0, \partial_{+}\right)$ such that

$$
\begin{array}{rlll}
-z_{n}<y_{n}^{-}, & z_{n}>y_{n}^{+}, & & \\
\partial_{p} H(x, p)>0 & \text { if } & \partial_{-} \leq x \leq-z_{n}, & p \geq q_{n}^{-}, \\
\partial_{p} H(x, p)<0 & \text { if } & z_{n} \leq x \leq \partial_{+}, & p \leq q_{n}^{+} .
\end{array}
$$

(49) and (50) (together with Assumption 4.1( (e)) imply that the complements of the sets $\left\{x: x \leq z_{n}\right\} \times\left[q_{n}^{-}, \infty\right)$ and $\left\{x: x \geq z_{n}\right\} \times\left(-\infty, q_{n}^{+}\right]$are preserved.

See Figure 4.1(b) for a picture.

Proof. Fix $n$. By Assumption 4.1 (d) we can choose $-z_{n}$ and $z_{n}$ in $\mathbb{K}^{\circ}$ such that

$$
\inf _{x \leq-z_{n}} \underset{p \in \mathbb{R}}{\operatorname{argmin}} H(x, p) \leq q_{n}^{-}, \quad \inf _{x \geq z_{n}} \underset{p \in \mathbb{R}}{\operatorname{argmin}} H(x, p) \geq q_{n}^{+} .
$$


By the assumed strict convexity of $p \mapsto H(x, p)$ we have $\partial_{p} H\left(x, p_{0}\right)=0$ for $p_{0}=$ $\operatorname{argmin}_{p \in \mathbb{R}} H(x, p)$ and $\partial_{p} H(x, p)<0$ for $p<p_{0}$ and $\partial_{p} H(x, p)>0$ for $p>p_{0}$. Therefore we conclude (49) and (50). We can choose $z_{n}$ large enough such that (48) is satisfied as well.

Remark 4.5. Note that Lemma 4.4 implies that for $x \in \mathbb{K}$ and $p \in \mathbb{R}$, one has the following implications for $t>0$

$$
\begin{array}{ccc}
\lim _{s \uparrow t} X_{t}^{x, p}=-1 & \Longrightarrow & \lim _{s \uparrow t} P_{t}^{x, p}=-\infty, \\
\lim _{s \uparrow t} X_{t}^{x, p}=1 & \Longrightarrow & \lim _{s \uparrow t} P_{t}^{x, p}=\infty .
\end{array}
$$

Note that in both cases $t=t_{x, p}$.

Lemma 4.6. Suppose that $(x, p) \in \mathbb{K} \times \mathbb{R}$ are such that $t_{x, p}<\infty$. Then

$$
\lim _{t \uparrow t_{x, p}}\left(X_{t}^{x, p}, P_{t}^{x, p}\right)
$$

takes its values in $\left\{\left(\partial_{-},-\infty\right),\left(\partial_{+}, \infty\right)\right\}$.

Proof. By [Per01, Theorem 2.4.3] (in case $\mathbb{K}=[-1,1]$, then applied to the Hamilton equations for the extended Hamiltonian as in Assumption 4.1(a)), for any compact set $K \subseteq \mathbb{K} \times \mathbb{R}$ there exists a time $\beta<t_{x, p}$ such that $\left(X_{\beta}^{x, p}, P_{\beta}^{x, p}\right) \notin K$. We will use this fact in two separate ways, depending on the setting, to prove the result.

First suppose $\mathbb{K}=[-1,1]$. Let $c=H(x, p)$ and $n \in \mathbb{N},(a, q)=\left(y_{n}^{+}, q_{n}^{+}\right)$and $(b, r)=\left(y_{n}^{-}, q_{n}^{-}\right)$(see Assumption 4.1(e)). By Lemma 4.3 the set (46) is compact. Hence there exists a $\beta_{0}<t_{x, p}$ such that $\left(X_{\beta_{0}}^{x, p}, P_{\beta_{0}}^{x, p}\right)$ is either in $\mathbb{k}_{y_{n}^{+}, q_{n}^{+}}$or $\rrbracket_{y_{n}^{-}, q_{n}^{-}}$. Assume it is in $\mathbb{K}_{y_{n}^{+}, q_{n}^{+}}$. Then $\left(X_{\beta_{0}}^{x, p}, P_{\beta_{0}}^{x, p}\right)$ is in $\mathbb{K}_{y_{n}^{+}, q_{n}^{+}}$for all $\beta \in\left(\beta_{0}, t_{x, p}\right)$ as $\mathfrak{y}_{y_{n}^{+}, q_{n}^{+}}$is preserved (see also Remark 4.2). In a similar way as above, for each $n \in \mathbb{N}$ there exists a $\beta_{n}$ such that $\left(X_{\beta}^{x, p}, P_{\beta}^{x, p}\right) \in K_{y_{n}^{+}, q_{n}^{+}}$for all $\beta>\beta_{n}$. This implies $\lim _{t \uparrow t_{x, p}}\left(X_{t}^{x, p}, P_{t}^{x, p}\right)=(1, \infty)$.

Now let $\mathbb{K}=\mathbb{R}$. Let $c=H(x, p)$ and let $n_{0} \in \mathbb{N}$ be such that $y_{n}^{-}<x<y_{n}^{+}$for $n \geq n_{0}$. Instead of using Lemma 4.3 in the above lines of proof for $\mathbb{K}=[-1,1]$, we can use Remark 4.2 and Lemma 4.4 to obtain the existence of a $\beta_{0}$ and $\beta_{n}$ as above and follow the same lines.

Proposition 4.7. Let $H$ satisfy Assumption 4.1. For all $u \in\left(0, \partial_{+}\right)$there exists a $v \in$ $\left(u, \partial_{+}\right)$such that for all $t \geq 0$ and all starting points and momenta $(x, p) \in(-v, v) \times \mathbb{R}$, with $X_{t}^{x, p}=b \in(-u, u)$ we have $X_{s}^{x, p} \in(-v, v)$ for all $s \in[0, t]$.

Proof. Let $n$ be such that $b \in\left(y_{n}^{-}, y_{n}^{+}\right)$. Let $z_{n}$ be as in Lemma 4.4 and $v>z_{n}$. Suppose $x \in(-v, v)$ and $p \in \mathbb{R}$ are such that $X_{t}^{x, p}=b$. As the end point $\left(X_{t}^{x, p}, P_{t}^{x, p}\right)$ lies outside the quadrants $\underline{y}_{y_{n}^{+}, q_{n}^{+}}$and $\mathbb{X}_{y_{n}^{-}, q_{n}^{-}}$the whole trajectory does not enter either of the quadrants as those quadrants are preserved.

As the complements of the sets $\left\{x: x \leq z_{n}\right\} \times\left[q_{n}^{-}, \infty\right)$ and $\left\{x: x \geq z_{n}\right\} \times\left(-\infty, q_{n}^{+}\right]$ are preserved, $X_{s}^{x, p}$ for $s \in[0, t]$ is prevented from entering $\{x: x \leq v\} \cup\{x: x \geq v\}$. 


\subsection{Additional model dependent conditions and properties}

In addition to the properties of the Hamiltonian flow that were established above, there are some peculiarities due to the boundaries of both settings that need to be treated separately. In the setting of the \pm 1 -space-model we need these auxiliary technical results in Section 4.3 below to show that optimizers that start at the boundary can be related to solutions of the Hamilton equations. For the $\mathbb{R}$-space-model, we introduce a condition that ensures that the rate function has compact sublevel sets, something that for the \pm 1 -space-model follows from Assumption 4.1 above.

\subsubsection{The \pm 1 -space-model}

In this section, we consider $\mathbb{K}=[-1,1]$. We start by showing that $\mathscr{L}$ is twice continuously differentiable on an appropriate domain. We then proceed by extending the regularity of the Hamiltonian flow up to the boundary of $[-1,1] \times \mathbb{R}$, after which we give an additional assumption that is needed to verify Proposition 4.14 (a) below.

Lemma 4.8. Let $\mathbb{K}=[-1,1]$ and let $H$ satisfy Assumption 4.1. Then $\mathscr{L}(-1, v)=$ $\infty$ for all $v \in\left(-\infty, v_{-}\right)$and $\mathscr{L}(1, v)=\infty$ for all $v \in\left(v_{+}, \infty\right)$, and $\mathscr{L}$ is $C^{2}$ on $(-1,1) \times \mathbb{R} \cup\left(\{-1\} \times\left(v_{-}, \infty\right)\right) \cup\left(\{1\} \times\left(-\infty, v_{+}\right)\right)$.

Proof. That $\mathscr{L}(-1, v)=\infty$ for all $v \in\left(-\infty, v_{-}\right)$and $\mathscr{L}(1, v)=\infty$ for all $v \in\left(v_{+}, \infty\right)$ follows from 44).

First we prove that $v \mapsto \mathscr{L}(-1, v)$ is $C^{2}$ on $\left(v_{-}, \infty\right)$, similarly one proves that $v \mapsto$ $\mathscr{L}(1, v)$ is $C^{2}$ on $\left(-\infty, v_{+}\right)$. Write $L(v)=\mathscr{L}(-1, v)$. In an analogous way as in CS04, Theorem A.2.3] one proves that for all $v \in\left(v_{-}, \infty\right)$ there exists a unique $p$ such that $L(v)=p v-H(-1, p)$, that $\lim _{v \rightarrow \infty} \frac{L(v)}{v}=\infty$, that $H(-1, \cdot)$ is the Legendre transform of $L$ (see the Fenchel-Moreau Theorem [RAS15, Chapter 5]) and that $p \in D^{-} L(v)$ if and only if $v \in D^{-} H(-1, p)$ (see [RAS15, Theorem 5.22]). With this one proves with the same argument as in the proof of [CS04, Theorem A.2.4] that $L$ is $C^{1}$ on $\left(v_{-}, \infty\right)$. Similarly, the argument of the proof of [CS04, Theorem A.2.5] carries over so that we have that $L$ is $C^{2}$ on $\left(v_{-}, \infty\right)$.

That $\mathscr{L}$ is $C^{2}$ on $(-1,1) \times \mathbb{R}$ follows from $[\mathrm{CS} 04$, Theorem A.2.7].

Following the lines of the proof of [CS04, Theorem A.2.7], it is sufficient to show that the map $q:(-1,1) \times \mathbb{R} \cup\left(\{-1\} \times\left(v_{-}, \infty\right)\right) \cup\left(\{1\} \times\left(-\infty, v_{+}\right)\right) \rightarrow \mathbb{R}$, which assigns to an element $(x, v)$ the unique $p$ such that $\mathscr{L}(x, v)=p v-H(x, p)$, is $C^{1}$. As $q$ satisfies $v-\partial_{p} H(x, q(x, v))=0$, and $\partial_{p}^{2} \bar{H}$ (see Assumption 4.1 (a) is $>0$ in an neighbourhood of $(x, p)$ for all $(x, p) \in[-1,1] \times \mathbb{R}$, by the implicit function theorem it follows that $q$ is $C^{1}$.

Lemma 4.9. Let $\mathbb{K}=[-1,1]$ and let $H$ satisfy Assumption 4.1 .

Suppose that $(\gamma, \eta):(0, t] \rightarrow[-1,1] \times \mathbb{R}$ is $C^{1}$ and satisfies the Hamilton equations (19). Then $(\gamma(0), \eta(0)):=\lim _{s \downarrow 0}(\gamma(s), \eta(s))$ exists in $[-1,1] \times \mathbb{R}$ and $(\gamma, \eta)$ is $C^{1}$ on $[0, t]$ and satisfies the Hamilton equations. 
Proof. Define the time-inverted trajectories $\gamma_{*}, \eta_{*}$ on $[0, t)$ by

$$
\gamma_{*}(s):=\gamma(t-s), \quad \eta_{*}(s):=\eta(t-s) .
$$

Then

$$
\left[\begin{array}{c}
\dot{\gamma_{*}}(s) \\
\dot{\eta}_{*}(s)
\end{array}\right]=\left[\begin{array}{c}
-\partial_{p} H\left(\gamma_{*}(s), \eta_{*}(s)\right) \\
\partial_{x} H\left(\gamma_{*}(s), \eta_{*}(s)\right)
\end{array}\right]
$$

Because $\left(\gamma_{*}, \eta_{*}\right)$ solves the time-inverted Hamilton equations, (53), Assumption 4.1(e) implies that if $n \in \mathbb{N}$ is such that $\left(\gamma_{*}(0), \eta_{*}(0)\right)$ is not in the set

$$
K_{y_{n}^{+}, q_{n}^{+}} \cup \rrbracket_{y_{n}^{-}, q_{n}^{-}}
$$

then $\left(\gamma_{*}(s), \eta_{*}(s)\right)$ is not in this set for all $s \in[0, t)$. As $H\left(\gamma_{*}(s), \eta_{*}(s)\right)=H\left(\gamma_{*}(0), \eta_{*}(0)=\right.$ : $c$ for all $s$, and the complement of (54) intersected with $H^{-1}(\{c\})$ is compact by Lemma 4.3 by $\left[\right.$ Per01, Theorem 2.4.3] we find that the maximal interval of existence $\left(\gamma_{*}, \eta_{*}\right)$ satisfying (53) is larger than $[0, t)$, which implies that both $\operatorname{limits}_{\lim } \lim _{s \downarrow 0}(\gamma(s), \eta(s))=$ $\lim _{s \uparrow t}\left(\gamma_{*}(s), \eta_{*}(s)\right)$ and $\lim _{s \downarrow 0}(\dot{\gamma}(s), \dot{\eta}(s))=-\lim _{s \uparrow t}\left(\dot{\gamma}_{*}(s), \dot{\eta}_{*}(s)\right)$ exist. Additionally, we conclude that the trajectory $(\gamma, \eta)$ solves the Hamilton equations on the interval $[0, t]$.

Assumption 4.10. Let $\mathbb{K}=[-1,1]$ and assume that $H$ satisfies Assumption 4.1 and that there is a function $S:[-1,1] \rightarrow[0, \infty]$ that is twice continuously differentiable on $(-1,1)$ such that

(a) $S$ is a Lyapunov function for $\dot{x}=\partial_{p} H(x, 0)$, i.e. if $x(t)$ solves $\dot{x}=\partial_{p} H(x, 0)$ then $t \mapsto S(x(t))$ is decreasing.

(b) $H\left(x, S^{\prime}(x)-p\right)=H(x, p)$ for all $(x, p) \in(-1,1) \times \mathbb{R}$.

(c) The map $x \mapsto \mathscr{L}(x, 0)$ is decreasing on a neighbourhood $U_{-1}$ of -1 in $[-1,1]$ and increasing on an neighbourhood $U_{1}$ of 1 in $[-1,1]$.

(d) We have

$$
\lim _{x \downarrow-1} \frac{\mathscr{L}(x, 0)-\mathscr{L}(-1,0)}{S(x)-S(-1)}=\infty, \quad \lim _{x \uparrow 1} \frac{\mathscr{L}(x, 0)-\mathscr{L}(1,0)}{S(x)-S(1)}=\infty .
$$

\subsubsection{The $\mathbb{R}$-space-model}

In this section, we consider $\mathbb{K}=\mathbb{R}$. We assume the existence of a Lyapunov function $\Upsilon$ that is needed to treat the non-compactness of $\mathbb{R}$ in the proof of compactness of the sublevel sets of the rate function (see Lemma 4.12).

Assumption 4.11. There is a continuously differentiable function $\Upsilon$ with compact sublevel sets, i.e. for all $c \in \mathbb{R}$ the set $\{x \in \mathbb{R}: \Upsilon(x) \leq c\}$ is compact, with the additional property that $\sup _{x \in \mathbb{R}} H\left(x, \Upsilon^{\prime}(x)\right)<\infty$. 


\subsection{The relation between optimizers and Hamiltonian trajectories and a study of the time-evolved rate function}

From the discussion above, we understand the behaviour of the Hamiltonian flow. In this section, we show that optimal trajectories exist (Lemma 4.12), and, combined with their dual trajectories, satisfy the Hamilton equations (Propositions 4.14 and 4.13 ). Then we show that the range of optimal trajectories can be controlled if $I_{0} \in C^{1, \partial}(\mathbb{K})$ (Proposition 4.15 .

Lemma 4.12. Let $H$ satisfy Assumption 4.1. If $\mathbb{K}=\mathbb{R}$, let $H$ in addition satisfy Assumption 4.11

Let $I_{0}$ have compact sublevel sets, then the rate function $\mathscr{I}$ in 13 has compact sublevel sets.

In addition, for every $t>0, a \in \mathbb{K}$ and $b \in \mathbb{K}^{\circ}$ there is an optimal trajectory $\gamma$ for $S_{t}(a, b)$ and $I_{t}(b)$.

Proof. Compactness of sublevel sets of $\mathscr{I}$ is generally proven as a part of a large deviation principle. For a proof of this result in the \pm 1 -space-model setting, see Kra16b, Theorem 2]. A similar proof for the $\mathbb{R}$-space-model can be carried out by using $\Upsilon$, see the proof of [CK17, Theorem A.14].

Pick $a \in \mathbb{K}, b \in \mathbb{K}^{\circ}$ and $t>0$. Pick a $C^{1}$ curve $\gamma$ connecting $a$ to $b$ in time $t$ such that in addition $(\gamma(s), \dot{\gamma}(s))$ takes its values in the region where $\mathscr{L}$ is $C^{2}$, cf. Lemma 4.8 for the case where $\mathbb{K}=[-1,1]$. This implies that $\gamma$ has finite cost. Thus, by the contraction principle and the compactness of the sublevel sets of $\mathscr{I}$, where we take as a starting rate function $I_{0}(a)=0$ and $I_{0}(x)=\infty$ if $x \neq a$, there is an optimizer for $S_{t}(a, b)$.

Again by the contraction principle, but now with a general starting rate function $I_{0}$, we find that there is an optimizer for $I_{t}(b)$.

Proposition 4.13. Let $H$ satisfy Assumption 4.1 in the setting that $\mathbb{K}=\mathbb{R}$, let $I_{0} \in$ $C^{1, \partial}(\mathbb{R})$, and $t>0$.

Let $a, b \in \mathbb{R}$ and suppose that $\gamma$ is an optimal trajectory for $S_{t}(a, b)$. Then $\gamma$ is $C^{2}$ on $[0, t]$. The dual trajectory $\eta$ defined on $[0, t]$ is $C^{1}$ and $(\gamma, \eta)$ satisfies the Hamilton equations $(19)$ on the interval $[0, t]$.

If $\gamma$ is an optimal trajectory for $I_{t}(b)$, then $\eta(0)=I_{0}^{\prime}(\gamma(0))$.

Proof. This follows by [CS04, Theorem 6.2.8 and 6.3.3].

Proposition 4.14. Let $H$ satisfy Assumption 4.10 in the setting that $\mathbb{K}=[-1,1]$, $I_{0} \in C^{1, \partial}[-1,1]$ and let $t>0$ and $a, b \in[-1,1]$.

(a) Any optimal trajectory $\gamma$ for $S_{t}(a, b)$ satisfies $\gamma(s) \in(-1,1)$ for all $s \in(0, t)$.

(b) Let $\gamma$ be an optimal trajectory for $S_{t}(a, b)$.

If $b \in(-1,1)$, then $\gamma$ is $C^{2}$ on $[0, t]$. The dual trajectory $\eta$ is defined on $[0, t]$ is $C^{1}$ and $(\gamma, \eta)$ satisfies the Hamilton equations (19) on the interval $[0, t]$. 
If $b \in\{-1,1\}$ then $\gamma$ is $C^{2}$ on $[0, t)$. The dual trajectory $\eta$ is defined on $[0, t)$ is $C^{1}$ and $(\gamma, \eta)$ satisfies the Hamilton equations 19$)$ on $[0, t)$ and $\lim _{s \uparrow t}(\gamma(s), \eta(s)) \in$ $\{(-1,-\infty),(1, \infty)\}$.

(c) Any optimal trajectory $\gamma$ for $I_{t}(b)$ satisfies $\gamma(0) \in(-1,1)$. Let $\eta$ be the dual trajectory as above, then we have $\eta(0)=I_{0}^{\prime}(\gamma(0))$.

Proof. (a) follows from Kra16a, Proposition 4.9] and Assumption 4.10

For (b), first we consider $b \in(-1,1)$. Let $\gamma$ be an optimizer for $S_{t}(a, b)$. By (a), we have $\gamma(s) \in(-1,1)$ for $s \in(0, t)$. Thus, for any $\delta \in(0, t)$, the trajectory $\gamma$ restricted to $[\delta, t]$ is contained in $(-1,1)$ and is the optimal trajectory over all absolutely continuous trajectories $\xi$ on $[\delta, t]$ with $\xi(t)=\gamma(t)$ and $\xi(\delta)=\gamma(\delta)$ of the functional

$$
\xi \mapsto \int_{\delta}^{t} \mathscr{L}(\xi(s), \dot{\xi}(s)) \mathrm{d} s .
$$

By Assumption 4.1 (a), (b) and [CS04, Theorem 6.2.8] (note that $\mathscr{L}$ is $C^{2}$ on $(-1,1) \times \mathbb{R}$ by Lemma 4.8, we find that the restriction of $\gamma$ to $[\delta, t]$ is $C^{2}$ and solves the EulerLagrange equation classically. This can be done for all $\delta>0$, so $\gamma$ is $C^{2}$ on $(0, t]$ and solves the Euler-Lagrange equation

$$
\frac{\mathrm{d}}{\mathrm{d} s} \partial_{v} \mathscr{L}(\gamma(s), \dot{\gamma}(s))=\partial_{x} \mathscr{L}(\gamma(s), \dot{\gamma}(s)), \quad s \in(0, t] .
$$

Let $\eta:(0, t] \rightarrow \mathbb{R}$ be the dual path as in (18) (note that $\mathscr{L}$ is $C^{2}$ by Lemma 4.8).

By [CS04, Theorem 6.3.3] $(\gamma, \eta)$ solves the Hamilton equations for all times $s \in(0, t]$. Lemma 4.9 extends this to the closed interval $[0, t]$.

If $b \in\{-1,1\}$, we can follow the same lines as above, now restricting to trajectories on $[\delta, t-\delta]$ instead of $[\delta, t]$ with $\delta \in\left(0, \frac{t}{2}\right)$ for example. For the $\operatorname{limit}_{s \uparrow t}(\gamma(s), \eta(s))$ we refer to Remark 4.5 .

For (c) suppose that there exists an optimal trajectory $\gamma$ such that $\gamma(0)=-1$ and $\gamma(t)=b$. The proof for $\gamma(0)=1$ is similar. By (a) and (b) there exists a $\kappa>0$ such that $\gamma(s)<1-\kappa$ for all $s \in\left[0, \frac{t}{2}\right]$.

Let $\rho \in C^{1}[0, t]$ be such that $\rho(0)>0, \rho=0$ on $\left[\frac{t}{2}, t\right]$ and such that $\rho$ takes its values in $[0, \kappa]$. Then $\gamma+\epsilon \rho$ attains its values in $[-1,1]$ for all $\epsilon \in[0,1]$.

We derive a contradiction by showing the existence of a $\epsilon \in(0,1)$ such that $\gamma+\epsilon \rho$ has a lower cost than $\gamma$, i.e., $\mathscr{J}(\gamma+\epsilon \rho)<\mathscr{J}(\gamma)$ (for $\mathscr{I}$ see (13)). We do this by showing that the difference quotient

$$
\frac{\mathscr{I}(\gamma+\varepsilon \rho)-\mathscr{I}(\gamma)}{\varepsilon}=\frac{I_{0}(\gamma(0)+\varepsilon \rho(0))-I_{0}(\gamma(0))}{\varepsilon}+\frac{J(\gamma+\varepsilon \rho)-J(\gamma)}{\varepsilon},
$$

converges to $-\infty$ as $\epsilon \downarrow 0$, where $J$ is the path-space cost

$$
J(\zeta):=\int_{0}^{t} \mathscr{L}(\zeta(s), \dot{\zeta}(s)) \mathrm{d} s .
$$


As $I_{0} \in C^{1, \partial}[-1,1]$, the first term on the right-hand-side of (56) converges to $-\infty$. Therefore it is sufficient to show that the second term on the right-hand-side is bounded from above for small $\epsilon$.

For $\theta>0$ we write

$$
\psi_{\theta}(s)=(\gamma(s)+\theta \rho(s), \dot{\gamma}(s)+\theta \dot{\rho}(s)) .
$$

By the mean-value theorem there exists a $\theta_{s} \in(0, \epsilon)$ for all $s \in[0, t]$ such that

$$
\frac{J(\gamma+\varepsilon \rho)-J(\gamma)}{\varepsilon}=\int_{0}^{t} \partial_{x} \mathscr{L}\left(\psi_{\theta_{s}}(s)\right) \rho(s)+\partial_{v} \mathscr{L}\left(\psi_{\theta_{s}}(s)\right) \dot{\rho}(s) \mathrm{d} s .
$$

The integrand and therefore the integral is bounded for all $\epsilon \leq \tilde{\epsilon}$ if

$$
\left\{\psi_{\theta}(s): s \in[0, t], \theta \in[0, \tilde{\epsilon}]\right\} \subseteq(-1,1) \times \mathbb{R} \cup\left(\{-1\} \times\left(v_{-}, \infty\right)\right) \cup\left(\{1\} \times\left(-\infty, v_{+}\right)\right),
$$

as the latter set is the domain on which $\partial_{x} \mathscr{L}$ and $\partial_{v} \mathscr{L}$ are continuous, see Lemma 4.8 . As $(\gamma, \eta)$ satisfies the Hamilton equations, we have $\dot{\gamma}(0)=\partial_{p} H(\gamma(0), \eta(0))>v_{-}$(by Assumption 4.1 (c) $)$. Hence we can choose $\tilde{\epsilon}$ small enough such that $\dot{\gamma}(0)+\theta \dot{\rho}(0)>v_{-}$ for all $\theta \in[0, \tilde{\epsilon}]$, which implies that (57) holds.

Now that it is established that $\gamma$ starts in the interior, we can apply CS04. Theorem 6.3.3] to obtain that $\eta(0)=I_{0}^{\prime}(\gamma(0))$.

Proposition 4.15. Let $H$ satisfy Assumption 4.1 and if $\mathbb{K}=[-1,1]$ additionally assume Assumption 4.10. Let $I_{0} \in C^{1, \partial}(\mathbb{K})$ and let $t>0$.

(a) For all $w \in\left(0, \partial_{+}\right)$there exists a $v \in\left(w, \partial_{+}\right)$such that for all $b \in[-w, w]$ and any optimal trajectory $\gamma$ for $I_{t}(b)$ we have $\gamma(s) \in[-v, v]$ for all $s \in[0, t]$.

(b) For all $m>0$ there exists a $v \in\left(0, \partial_{+}\right)$such that for all $b \in\left(\partial_{-},-v\right) \cup\left(v, \partial_{+}\right)$and any optimal trajectory $\gamma$ for $I_{t}(b)$ with dual trajectory $\eta$

$$
\begin{aligned}
\eta(t) \geq m \quad & \text { if } \quad b \in\left(v, \partial_{+}\right), \\
\eta(t) \leq-m & \text { if } \quad b \in\left(\partial_{-},-v\right) .
\end{aligned}
$$

Proof. (a) follows from Proposition 4.7 together with Proposition $4.13($ for $\mathbb{K}=\mathbb{R}$ ) or together with Proposition 4.14)(b) (for $\mathbb{K}=[-1,1])$.

(b) Fix $n$ such that $q_{n}^{+} \geq m$ and $q_{n}^{-} \leq-m$. As $I_{0} \in C^{1, \partial}(\mathbb{K})$, there is a $v \in\left(0, \partial_{+}\right)$ such that

$$
\sup _{x \in\left(\partial_{-},-v\right)} I_{0}^{\prime}(x) \leq q_{n}^{-}, \quad \inf _{x \in\left(v, \partial_{+}\right)} I_{0}^{\prime}(x) \geq q_{n}^{+},
$$

Let $z_{n}$ be as in Lemma 4.4. We may and do assume that $v>z_{n}$.

Let $b>v$ and $\gamma$ the optimal trajectory for $I_{b}(b)$ and $\eta$ its dual trajectory. We show that $\eta(t) \geq y_{n}^{+}$(in a similar way one proves that $b<-v$ implies $\eta(t) \leq y_{n}^{-}$).

Suppose that $\gamma(0)>v$. Then $I_{0}^{\prime}(\gamma(0))>q_{n}^{+}$by (58). As $\gamma(0)>1-\delta>1-\delta_{n}>y_{n}^{+}$ (see 48),$(\gamma, \eta)$ starts and, therefore, stays in the quadrant $\mathfrak{y}_{y_{n}^{+}, q_{n}^{+}}$, which implies $\eta(t) \geq q_{n}^{+}$. 
Suppose that $\gamma(0) \leq v$. Because the complement of the region $\{x: x \geq v\} \times\left(-\infty, q_{n}^{+}\right]$ is preserved (by Lemma 4.4), the Hamiltonian trajectory $(\gamma, \eta)$ cannot enter this region. Because $\gamma(t)=b \geq v>z_{n} \geq y_{n}^{+}$, this implies that $\eta(t) \geq q_{n}^{+}$.

\section{Properties of the time-evolved rate function}

To rigorously study the time-dependent rate function $u(t, x):=I_{t}(x)$ and $x \mapsto I_{t}(x)$ for both the \pm 1 -space-model and the $\mathbb{R}$-space-model, we establish local semi-concavity and the boundary behaviour. The local semi-concavity implies we can use sub-gradients as in the classical theory and conclude that the push-forward of the gradient of the starting rate function under the Hamiltonian flow contains the reachable gradients of $I_{t}$.

Lemma 5.1. Assume Assumption 2.10. Then $I_{t}$ is locally semi-concave on $\mathbb{K}^{\circ}$ for all $t \geq 0$. Moreover, $(t, x) \mapsto I_{t}(x)=u(t, x)$ is locally semi-concave on $(0, \infty) \times \mathbb{K}^{\circ}$.

Proof. Let $w \in\left(0, \partial_{+}\right)$and $v \in\left(w, \partial_{+}\right)$be as in Proposition 4.15. Let $x, y \in \mathbb{K}^{\circ}$ be such that $x<y,[x, y] \subseteq[-w, w]$ and $q:=v+y-x<\partial_{+}$. We show that $I_{t}$ is semi-concave on $[x, y]$ (this is sufficient as for all $z \in \mathbb{K}^{\circ}$ there exist $w, x, y$ as above with $z \in[x, y]$ ).

Let $\lambda \in[0,1]$ and $\xi$ be an optimal trajectory for $I_{t}(\lambda x+(1-\lambda) y)$. By the choice of $v$, we have that $\xi$ attains its values in $[-v, v]$. In addition, consider the trajectories $\xi_{x}, \xi_{y}$ defined by

$$
\xi_{x}(s)=\xi(s)+\frac{s}{t}(1-\lambda)(x-y), \quad \xi_{y}(s)=\xi(s)+\frac{s}{t} \lambda(y-x) .
$$

Note that $\xi_{x}(t)=x$ and $\xi_{y}(t)=y, \lambda \xi_{x}+(1-\lambda) \xi_{y}=\xi$ and $\xi_{x}(0)=\xi(0)=\xi_{y}(0)$. The trajectories $\xi_{x}, \xi_{y}$ and $\xi$ take their values in $[-q, q]$. As $\mathscr{L}$ is $C^{2}$ on $\mathbb{K}^{\circ} \times \mathbb{R}$ by Lemma 4.8. we find that $\mathscr{L}$ is semi-concave on $[-q, q] \times \mathbb{R}$. Thus the first statement follows similarly as in the proof of [CS04, Theorem 6.4.3]:

$$
\begin{aligned}
& \lambda I_{t}(x)+(1-\lambda) I_{t}(y)-I_{t}(\lambda x+(1-\lambda) y) \\
& \leq \int_{0}^{t} \lambda \mathscr{L}\left(\xi_{x}(s), \dot{\xi}_{x}(s)\right)+(1-\lambda) \mathscr{L}\left(\xi_{y}(s), \dot{\xi}_{y}(s)\right)+\mathscr{L}(\xi(s), \dot{\xi}(s)) \mathrm{d} s \\
& \leq \int_{0}^{t} C \frac{\lambda(1-\lambda)}{2} \frac{s^{2}+1}{t^{2}}\left((1-\lambda)^{2}+\lambda^{2}\right)|y-x|^{2} \mathrm{~d} s=2 C\left(\frac{t}{3}+\frac{1}{t}\right) \frac{\lambda(1-\lambda)}{2}|x-y|^{2} .
\end{aligned}
$$

The 'moreover' statement follows along the same lines as the proof of CS04, Corollary 6.4.4].

Proposition 5.2. Assume assumption 2.10. Let $t>0$.

(a) Fix $b \in \mathbb{K}^{\circ}$ and let $\gamma$ be an optimizer for $I_{t}(b)$ and let $\eta$ be the dual trajectory, then $\eta(t) \in D^{+} I_{t}(b)$.

(b) For any $b \in \mathbb{K}^{\circ}$ the map that associates to $\left(p_{t}, p\right) \in D^{*} u(t, b)$ the Hamiltonian trajectory $(\gamma, \eta)$ with terminal condition $\gamma(t)=b$ and $\eta(t)=p$ provides a one-toone correspondence between $D^{*} u(t, b)$ and optimizers of $I_{t}(b)$. 
(c) We have

$$
\left\{(x, p) \mid x \in \mathbb{K}^{\circ}, p \in D^{*} I_{t}(x)\right\} \subseteq \mathscr{G}_{t} .
$$

For any $b \in \mathbb{K}^{\circ}$ and any element $p \in D^{*} I_{t}(b)$, the Hamiltonian trajectory $(\gamma, \eta)$ with terminal condition $\gamma(t)=b$ and $\eta(t)=p$ yields an optimal trajectory $\gamma$ for $I_{t}(b)$.

Proof. (a) and (b) can be proven as [CS04, Theorems 6.4.8 and 6.4.9] using that optimizers are bounded away from the boundary by Proposition 4.15 (a).

The proof of (c) uses a variation on the ideas in the proof of [CS04, Theorem 6.4.9]. Let $x \in \mathbb{K}^{\circ}$ and $p \in D^{*} I_{t}(x)$. By definition, there are $\left(x_{k}, p_{k}\right) \in \mathbb{K}^{\circ} \times \mathbb{R}$ such that $\left(x_{k}, p_{k}\right) \rightarrow(x, p), I_{t}^{\prime}$ is differentiable at $x_{k}$ and $p_{k}=I_{t}^{\prime}\left(x_{k}\right)$.

Let $\gamma_{k}:[0, t] \rightarrow \mathbb{K}$ be an optimizing trajectory for $I_{t}\left(x_{k}\right)$ and let $\eta_{k}$ be the dual trajectory. By Propositions 4.13 and $4.14\left(\gamma_{k}, \eta_{k}\right)$ satisfies Hamilton's equations and $\eta_{k}(0)=I_{0}^{\prime}\left(\gamma_{k}(0)\right)$. CS04, Theorem 6.4.8] implies that $\left(\gamma_{k}(t), \eta_{k}(t)\right)=\left(x_{k}, p_{k}\right)$. By continuous dependence on the final conditions, we have $\left(\gamma_{k}(s), \eta_{k}(s)\right) \rightarrow(\gamma(s), \eta(s))$ for all $s \in[0, t]$ (see Per01, Theorem 2.3.2]), where $(\gamma, \eta)$ solves the Hamilton equations with $(\gamma(t), \eta(t))=(x, p)$. By continuity of $\partial_{p} H$, we also obtain $\dot{\gamma}_{k}(s) \rightarrow \dot{\gamma}(s)$ for all $s \in[0, t]$. We obtain that indeed $(x, p) \in \mathscr{G}_{t}$. By continuity of $I_{0}, I_{t}$ and $\mathscr{L}$ we find additionally that

$$
\begin{aligned}
I_{t}(x) & =\lim _{k \rightarrow \infty} I_{t}\left(x_{k}\right) \\
& =\lim _{k \rightarrow \infty} I_{0}\left(\gamma_{k}(0)\right)+\int_{0}^{t} \mathscr{L}\left(\gamma_{k}(s), \dot{\gamma}_{k}(s)\right) \mathrm{d} s \\
& =I_{0}(\gamma(0))+\int_{0}^{t} \mathscr{L}(\gamma(s), \dot{\gamma}(s)) \mathrm{d} s,
\end{aligned}
$$

thus $\gamma$ is an optimizer for $I_{t}(x)$.

Lemma 5.3. Assume Assumption 2.10. Then

$$
\sup _{a \in\left(0, \partial_{+}\right)} \inf _{b \geq a} \inf D^{+} I_{t}(b)=\infty, \quad \inf _{a \in\left(\partial_{-}, 0\right)} \sup _{b \leq a} \sup D^{+} I_{t}(b)=-\infty .
$$

Proof. First note that $D^{+} I_{t}(b)=\operatorname{co} D^{*} I_{t}(b)$. By Proposition 5.2 (c) $p \in D^{*} I_{t}(b)$ corresponds to a trajectory $(\gamma, \eta)$ that solves the Hamilton equations with terminal condition $(\gamma(t), \eta(t))=(b, p)$, the rest follows by Proposition 4.15 (b).

We end this section with the proof of the statement that $u$ is a viscosity solution of the Hamilton-Jacobi equation (23).

Proof of Proposition 2.14. The proof follows as in the proof of [CS04, Theorem 6.4.5], using Proposition 4.15 (a) to make sure that optimal trajectories remain in compact sets bounded away from the boundary and Lemma 5.1 to make sure that $u$ is locally semi-concave on $\mathbb{K}^{\circ} \times(0, \infty)$. 


\section{Topological structure of $\mathscr{G}_{t}$}

In this section we study the structure of $\mathscr{G}_{t}$. Inspired by the result of Proposition 4.6 we introduce an extension of the Hamiltonian flow to include the points $\left(\partial_{-},-\infty\right)$ and $\left(\partial_{+}, \infty\right)$. In this way we make sense of Hamilton paths starting at $(x, p)$ for times $t>t_{x, p}$. In addition, we extend $\mathscr{G}$ to include the points at infinity and thus we can use the properties of connected sets to study the structure of $\mathscr{G}_{t}$ in Proposition 6.2.

Proposition 6.1. Assume assumption 2.10, Let

$$
E:=\left\{(t, x, p) \in[0, \infty) \times \mathbb{K} \times \mathbb{R}: t<t_{x, p}\right\} .
$$

We extend the space $\mathbb{K} \times \mathbb{R}$ with two points and write $\mathfrak{S}:=\mathbb{K} \times \mathbb{R} \cup\left\{\left(\partial_{-},-\infty\right)\right\} \cup\left\{\left(\partial_{+}, \infty\right)\right\}$. We equip $\mathfrak{S}$ with the topology generated by the open subsets of $\mathbb{K} \times \mathbb{R}$ together with the sets $\mathbb{K}_{a, b}^{\circ} \cup\left\{\left(\partial_{+}, \infty\right)\right\}$ and $\mathbb{T}_{a, b}^{\circ} \cup\left\{\left(\partial_{-},-\infty\right)\right\}$ for $a \in \mathbb{K}^{\circ}$ and $b \in \mathbb{R}$.

(a) The map $(x, p) \mapsto t_{x, p}$ is lower semi-continuous and $E \mapsto \mathbb{K} \times \mathbb{R},(t, x, p) \mapsto$ $\left(X_{t}^{x, p}, P_{t}^{x, p}\right)$ is continuous.

(b) The map $\Psi:[0, \infty) \times \mathfrak{S} \rightarrow \mathfrak{S}$ defined by ${ }^{5}$

$$
\Psi(t, x, p):= \begin{cases}\left(X_{t}^{x, p}, P_{t}^{x, p}\right) & \text { if }(t, x, p) \in E, \\ \lim _{s \uparrow t_{x, p}\left(X_{s}^{x, p}, P_{s}^{x, p}\right)} \text { if }(x, p) \in \mathbb{K} \times \mathbb{R}, t \geq t_{x, p}, \\ \left(\partial_{-},-\infty\right) & \text { if }(x, p)=\left(\partial_{-},-\infty\right) \\ \left(\partial_{+}, \infty\right) & \text { if }(x, p)=\left(\partial_{+}, \infty\right) .\end{cases}
$$

is continuous.

Proof. (a) follows from [Per01, Theorem 2.5.1] or [Tes12, Theorem 2.8] for example (as those theorems apply to open sets, in case $\mathbb{K}=[-1,1]$ consider $(-1-\epsilon, 1+\epsilon)$ for $\epsilon>0$ and $\hat{H}$ as in Assumption 4.1 (a) instead of $K$ and $H$ ).

(b) The continuity on $E$ follows from (a) The continuity of $\Psi$ at $\left(t, \partial_{-},-\infty\right)$ and $\left(t, \partial_{+}, \infty\right)$ follows by the preservation of quadrants, as mentioned in Remark 4.2 and Remark 4.5. Therefore we are left to prove continuity of $\Psi$ at $(t, x, p)$ with $t \geq t_{x, p}$. We may restrict ourselves to sequential continuity because the topology on $\mathfrak{S}$ is metrizable by Urysohn's metrization theorem (e.g. Run05, Theorem 4.1.10]); the topology is second countable and normal (we leave it to the reader to check those properties).

Assume $(t, x, p)$ is such that $t \geq t_{x, p}$ and $\Psi(t, x, p)=\left(\partial_{+}, \infty\right)$ (the case $\Psi(t, x, p)=$ $\left(\partial_{-},-\infty\right)$ is similar). Suppose that $\left(x_{n}, p_{n}, t_{n}\right) \rightarrow(x, p, t)$.

It is sufficient to show that for all $k \in \mathbb{N}$ there exists an $N$ such that for all $n \geq N$

$$
\left(X_{t_{n}}^{x_{n}, p_{n}}, P_{t_{n}}^{x_{n}, p_{n}}\right) \in K_{y_{k}, q_{k}} \cup\left\{\left(\partial_{+}, \infty\right)\right\} .
$$

Let $k \in \mathbb{N}$ and $s<t_{x, p}$ be such that

$$
\left(X_{s}^{x, p}, P_{s}^{x, p}\right) \in K_{y_{k}, q_{k}} .
$$

\footnotetext{
${ }^{5}$ Note that $\lim _{s \uparrow t_{x, p}}\left(X_{s}^{x, p}, P_{s}^{x, p}\right)$ is welldefined by Lemma 4.6
} 
By (a) we have $\liminf _{n \rightarrow \infty} t_{x_{n}, p_{n}} \geq t_{x, p}$. Hence there exists a $N_{0}$ such that $t_{x_{n}, p_{n}}>s$ for all $n \geq N_{0}$. As $s<t_{x_{n}, p_{n}}$ and $s<t_{x, p}$ we have by (a)

$$
\lim _{n \rightarrow \infty}\left(X_{s}^{x_{n}, p_{n}}, P_{s}^{x_{n}, p_{n}}\right)=\left(X_{s}^{x, p}, P_{s}^{x, p}\right) \in K_{y_{k}, q_{k}} .
$$

Therefore there exists an $N_{1}>N_{0}$ such that $\left(X_{s}^{x_{n}, p_{n}}, P_{s}^{x_{n}, p_{n}}\right) \in \mathbb{K}_{y_{k}, q_{k}}$ for all $n \geq N_{1}$. Let $N>N_{1}$ be such that $t_{n}>s$ for all $n \geq N$. As $K_{y_{k}, q_{k}}$ is preserved under the Hamiltonian flow and $t_{n}>s$ for $n \geq N$ we obtain that (59) holds for all $n \geq N$.

Proposition 6.2. Assume Assumption 2.10. Define $E_{t}:=\left\{x \in \mathbb{K}: t_{x, I_{0}^{\prime}(x)}>t\right\}$ and let $\Phi_{t}: E_{t} \rightarrow \mathbb{K} \times \mathbb{R}$ be defined by $\Phi_{t}(x):=\left(X_{t}^{x, I_{0}^{\prime}(x)}, P_{t}^{x, I_{0}^{\prime}(x)}\right)$. Note that $\mathscr{G}_{t}=\Phi_{t}\left(E_{t}\right)$.

(a) $E_{t}$ is open for all $t, \Phi_{t}$ is continuous and injective on $E_{t}$.

(b) Let $t>0$ and suppose that $(a, b) \subseteq E_{t}, a, b \notin E_{t}$. Then $\Phi_{t}(a, b)$ is path-connected and both $\lim _{z \downarrow a} \Phi_{t}(z)$ and $\lim _{z \uparrow b} \Phi_{t}(z)$ are in $\left\{\left(\partial_{-},-\infty\right),\left(\partial_{+}, \infty\right)\right\}$.

(c) If $(a, b),(c, d)$ are consecutive maximally (as in (b) connected components in $E_{t}$, then $\lim _{z \uparrow b} \Phi_{t}(z)=\lim _{z \downarrow c} \Phi_{t}(z)$.

(d) Let

$$
z_{-}:=\inf \left\{z \in \mathbb{K}^{\circ} \mid z \in E_{t}\right\}, \quad z_{+}:=\sup \left\{z \in \mathbb{K}^{\circ} \mid z \in E_{t}\right\} .
$$

Then $\lim _{z \downarrow z_{-}} \Phi_{t}(z)=\left(\partial_{-},-\infty\right)$ and $\lim _{z \uparrow z_{+}} \Phi_{t}(z)=\left(\partial_{+}, \infty\right)$ for all $t>0$.

(e) There exists a maximal (as in (b)) connected component $(a, b)$ in $E_{t}$ that connects $\left(\partial_{-},-\infty\right)$ to $\left(\partial_{+}, \infty\right)$, i.e., $\lim _{z \downarrow a} \Phi_{t}(z)=\left(\partial_{-},-\infty\right)$ and $\lim _{z \uparrow b} \Phi_{t}(z)=\left(\partial_{+}, \infty\right)$.

(f) Let $\gamma_{t}: E_{t} \rightarrow \mathbb{K}$ be defined by $\gamma_{t}(x):=X_{t}^{x, I_{0}^{\prime}(x)}$. $\gamma_{t}$ is strictly increasing on $E_{t}$ if and only if $\mathscr{G}_{t}$ is a graph.

Proof. Note that $I_{0}^{\prime}$ is continuous and that $\Phi_{t}(x)=\Psi\left(t, x, I_{0}^{\prime}(x)\right)$ for $x \in E_{t}$, with $\Psi$ as in Proposition 6.2. By this one deduces (a), (b) and (c). (d) follows from preservation of quadrants and the fact that $I_{0} \in C^{1, \partial}(\mathbb{K})$. (e) then follows as $\mathbb{K} \rightarrow \mathfrak{S}$ given by $x \mapsto \Psi\left(t, x, I_{0}^{\prime}(x)\right)$ is continuous and connects $\left(\partial_{-},-\infty\right)$ with $\left(\partial_{+}, \infty\right)$ by (d)] (f) follows from the previous.

Remark 6.3. By Proposition 6.2(e), we see that if $E_{t}$ has at least two disconnected components, then $\mathscr{G}_{t}$ has an overhang at an $x_{0}$. But in this situation, generally one cannot find $x_{1}, x_{2}$ at which $\mathscr{G}_{t}$ has no overhang, with $x_{1}<x_{0}<x_{2}$, so that we cannot apply Proposition 2.16 to conclude that $I_{t}$ is not differentiable.

\section{Dynamics that preserve order}

In this section, we give the proof of Theorem 3.5 and give sufficient conditions on $H$ for it to preserve order (in Proposition 7.2, see Definition 3.2)(c)). First we prove in Lemma 7.1 that if the order of the momentum is preserved along some time interval, that the order of the position is also preserved. 
Lemma 7.1. Assume Assumption 2.10. Consider two pairs $\left(x_{1}, p_{1}\right),\left(x_{2}, p_{2}\right) \in \mathbb{K} \times \mathbb{R}$ and a time $t^{*} \in[0, \infty]$ with $t^{*} \leq t_{x_{1}, p_{1}} \wedge t_{x_{2}, p_{2}}$ such that $x_{1}<x_{2}$ and $P_{t}^{x_{1}, p_{1}}<P_{t}^{x_{2}, p_{2}}$ for all $0<t<t^{*}$. Then $X_{t}^{x_{1}, p_{1}}<X_{t}^{x_{2}, p_{2}}$ for all $t<t^{*}$.

Proof. Assume the contrary, that there is some $t<t^{*}$ such that $X_{t}^{x_{1}, p_{1}} \geq X_{t}^{x_{2}, p_{2}}$. Then there exists a $0<t_{0}<t^{*}$ such that

$$
X_{t}^{x_{1}, p_{1}}<X_{t}^{x_{2}, p_{2}} \text { for all } t<t_{0} \text { and } X_{t_{0}}^{x_{1}, p_{1}}=X_{t_{0}}^{x_{2}, p_{2}}=a .
$$

Therefore $\partial_{p} H\left(a, P_{t_{0}}^{x_{1}, p_{1}}\right)=\dot{X}_{t_{0}}^{x_{1}, p_{1}} \geq \dot{X}_{t_{0}}^{x_{2}, p_{2}}=\partial_{p} H\left(a, P_{t_{0}}^{x_{2}, p_{2}}\right)$ from which we conclude, by strict monotonicity of $\partial_{p} H$ in the $p$ variable that $P_{t_{0}}^{x_{1}, p_{1}} \geq P_{t_{0}}^{x_{2}, p_{2}}$ contradicting our assumption.

Proposition 7.2. Assume Assumption 2.10. Suppose that $A \subseteq \mathbb{K} \times \mathbb{R}$ is preserved under $H$ and that either (i) or (ii) holds:

(i) for all $p$, we have $x \mapsto \partial_{x} H(x, p)$ is strictly decreasing on $\{x:(x, p) \in A\}$.

(ii) the map $(x, p) \mapsto \partial_{x} H(x, p)$ is decreasing in both coordinates on the set $A$.

Then $H$ preserves order on $A$.

Proof. Assume the contrary, that there is some $s<t_{x_{1}, p_{1}} \wedge t_{x_{2}, p_{2}}$ such that $X_{s}^{x_{1}, p_{1}} \geq$ $X_{s}^{x_{2}, p_{2}}$ or $P_{s}^{x_{1}, p_{1}} \geq P_{s}^{x_{2}, p_{2}}$. We assume that $t_{0}$ is the smallest of such times $s$. Then we have exactly one of the following two situations as the Hamiltonian trajectories do not meet:

(a) $P_{t}^{x_{1}, p_{1}}<P_{t}^{x_{2}, p_{2}}$ for all $t \leq t_{0}$ and

$$
X_{t}^{x_{1}, p_{1}}<X_{t}^{x_{2}, p_{2}} \text { for all } t<t_{0} \text { and } X_{t_{0}}^{x_{1}, p_{1}}=X_{t_{0}}^{x_{2}, p_{2}}=a .
$$

(b) $X_{t}^{x_{1}, p_{1}}<X_{t}^{x_{2}, p_{2}}$ for all $t \leq t_{0}$ and

$$
P_{t}^{x_{1}, p_{1}}<P_{t}^{x_{2}, p_{2}} \text { for all } t<t_{0} \text { and } P_{t_{0}}^{x_{1}, p_{1}}=P_{t_{0}}^{x_{2}, p_{2}}=q .
$$

If (a) holds then we have a contradiction by Lemma 7.1. If (b) holds we first proceed by using variant $(i)$. As in the proof of the lemma above, we find $-\partial_{x} H\left(X_{t_{0}}^{x_{1}, p_{1}}, p\right)=$ $\dot{P}_{t_{0}}^{x_{1}, p_{1}} \geq \dot{P}_{t_{0}}^{x_{2}, p_{2}}=-\partial_{x} H\left(X_{t_{0}}^{x_{2}, p_{2}}, p\right)$ which by the assumed strict monotonicity of $\partial_{x} H$ on $A$ implies that $X_{t_{0}}^{x_{1}, p_{1}}>X_{t_{0}}^{x_{2}, p_{2}}$. This is in contradiction with (b).

Next, we use variant (ii). We find

$$
p_{2}-p_{1}=P_{0}^{x_{2}, p_{2}}-P_{0}^{x_{1}, p_{1}}=\int_{0}^{t_{0}} \partial_{x} H\left(X_{s}^{x_{2}, p_{2}}, P_{s}^{x_{2}, p_{2}}\right)-\partial_{x} H\left(X_{s}^{x_{1}, p_{1}}, P_{s}^{x_{1}, p_{1}}\right) \mathrm{d} s .
$$

By assumption, the left-hand-side is strictly greater than 0 . For $0 \leq s \leq t_{0}$, however, we have $X_{s}^{x_{1}, p_{1}}<X_{s}^{x_{2}, p_{2}}$ and $P_{s}^{x_{1}, p_{1}}<P_{s}^{x_{2}, p_{2}}$, which by (ii) is non-positive. 
Proof of Theorem 3.5. For both (a) and (b) we use the following for $t>0$. By Proposition 6.2(f) it follows that if $\gamma_{t}$ is strictly increasing, then $\mathscr{G}_{t}$ is a graph and thus $I_{t} \in C^{1, \partial}(\mathbb{K})$ by Theorem 2.13 .

Theorem 3.5)(a) follows then immediately from Proposition 7.2 as convexity of a differentiable function implies that its derivative is increasing.

Theorem 3.5(b). Let $a, b>0, a \in \mathbb{K}^{\circ}$ be such that $H$ preserves order on $\mathbb{\lambda}_{-a,-b \text {, and }}$ on $\nVdash_{a, b}, I_{0}^{\prime}([-a, a]) \subseteq[-b, b]$ and $I_{0}$ is convex on $\left(\partial_{-},-a\right]$ and on $\left[a, \partial_{+}\right)$.

That $\gamma_{t}$ is strictly increasing outside $(-a, a)$ follows by the assumed strict convexity of $I_{0}$ and that $H$ preserves order by using Proposition 7.2. We will show that $\gamma_{t}$ is differentiable and that $\gamma_{t}^{\prime}(x)>0$ for all $x \in[-a, a]$ and all $t$ small enough.

As $[-a, a] \times[-b, b]$ is compact, by Proposition 6.1 (a) there exists a $t^{*}>0$ such that $U:=\left(0, t^{*}\right) \times(-a, a) \times(-b, b) \subseteq E$. By [Per01, Theorem 2.5.1] the map $\Psi$ is $C^{1}$ on $U$. As $x \mapsto\left(x, I_{0}^{\prime}(x)\right)$ is $C^{1}$ by assumption, the map $(t, x) \mapsto \Psi\left(t, x, I_{0}^{\prime}(x)\right)$ is $C^{1}$ on $\left(0, t^{*}\right) \times[-a, a]$. By definition $\gamma_{t}(x)$ is the first coordinate of $\Psi\left(t, x, I_{0}^{\prime}(x)\right)$ and therefore $(t, x) \mapsto \gamma_{t}(x)$ is also $C^{1}$ on $\left(0, t^{*}\right) \times[-a, a]$.

Note that $t \mapsto \inf _{x \in[-a, a]} \gamma_{t}^{\prime}(x)$ is continuous and equal to 1 for $t=0$. Therefore there exists a $0<t_{0} \leq t^{*}$ such that $\gamma_{t}^{\prime}(x)>0$ for all $0<t<t_{0}$ and $x \in[-a, a]$.

\section{Occurrence of non-differentiability}

In this section, we introduce the methods necessary for the proofs of Theorem 3.6 .

\subsection{Linearization around stationary points}

We start by studying linearizations of the Hamiltonian flows around stationary points. In contrast to homeomorphisms between flows, see e.g. [Per01], $C^{1}$ diffeomorphisms are difficult to construct. We will refer to GHR03 for one such construction.

Theorem 8.1. GHR03, Theorem 3] Let $H$ satisfy Assumption 2.10 and be $C^{\infty}$. Assume that $x_{0}$ is a stationary point and that $m \neq 0$ ( $m$ as in $(26)$ ). Then $H$ admits a $C^{1}$ linearization at $\left(x_{0}, 0\right)$.

Proof of Theorem 3.6 (a). We show that $\gamma_{t}^{\prime}\left(x_{0}\right)<0$, which establishes an overhang at $x_{0}$ by Proposition 6.2(f)

We first establish this for the case that $m \neq 0$. The linearized system (25) with $\left(\xi_{x}(0), \zeta_{x}(0)\right):=\left(x, x I_{0}^{\prime \prime}\left(x_{0}\right)\right)$ is solved by

$$
\left[\begin{array}{l}
\xi_{x}(t) \\
\zeta_{x}(t)
\end{array}\right]=\exp \left(t\left[\begin{array}{cc}
m & c \\
0 & -m
\end{array}\right]\right)\left[\begin{array}{c}
x \\
x I_{0}^{\prime \prime}\left(x_{0}\right)
\end{array}\right]=x\left[\begin{array}{c}
e^{m t}+\frac{c}{2 m}\left(e^{m t}-e^{-m t}\right) I_{0}^{\prime \prime}\left(x_{0}\right) \\
e^{-m t} I_{0}^{\prime \prime}\left(x_{0}\right)
\end{array}\right] .
$$

$e^{m t}+\frac{c}{2 m}\left(e^{m t}-e^{-m t}\right) I_{0}^{\prime \prime}\left(x_{0}\right)<0$ if $t>t_{0}$, whence $\left.\partial_{x} \xi_{x}(t)\right|_{x=x_{0}}<0$. Denote by $\Theta$ the first component of $\Psi^{-1}$, the inverse of the linearization (see Definition 3.3). As $D \Psi\left(x_{0}, 0\right)=\mathbb{1}$, the identity matrix, by the inverse function theorem we have $D \Psi^{-1}\left(x_{0}, 0\right)=\mathbb{1}$ and thus 
$\partial_{\xi} \Theta\left(x_{0}, 0\right)=1$ and $\partial_{\zeta} \Theta\left(x_{0}, 0\right)=0$. Therefore

$$
\begin{aligned}
\gamma_{t}^{\prime}\left(x_{0}\right)=\partial_{\xi} \Theta & \left.\left(\xi_{x_{0}}(t), \zeta_{x_{0}}(t)\right) \partial_{x} \xi_{x}(t)\right|_{x=x_{0}} \\
& +\left.\partial_{\zeta} \Theta\left(\xi_{x_{0}}(t), \zeta_{x_{0}}(t)\right) \partial_{x} \zeta_{x}(t)\right|_{x=x_{0}}<0
\end{aligned}
$$

In case $m=0$, the linearized system 25 with $\left(\xi_{x}(0), \zeta_{x}(0)\right):=\left(x, x I_{0}^{\prime \prime}\left(x_{0}\right)\right)$ is solved by

$$
\left[\begin{array}{l}
\xi_{x}(t) \\
\zeta_{x}(t)
\end{array}\right]=\exp \left(t\left[\begin{array}{ll}
0 & c \\
0 & 0
\end{array}\right]\right)\left[\begin{array}{c}
x \\
x I_{0}^{\prime \prime}\left(x_{0}\right)
\end{array}\right]=x\left[\begin{array}{c}
1+t c I_{0}^{\prime \prime}\left(x_{0}\right) \\
I_{0}^{\prime \prime}\left(x_{0}\right)
\end{array}\right] .
$$

As $1+t c I_{0}^{\prime \prime}\left(x_{0}\right)<0$ for $t>t_{0}$, again we obtain $\partial_{x} \xi_{x}(t)<0$ so that the result follows as for the case $m \neq 0$.

Remark 8.2. Note that we actually proved that $\left\{y \in \mathbb{R}:\left(x_{0}, y\right) \in \mathscr{G}_{t}\right\} \geq 3$ has at least three elements.

\subsection{Rotating areas in the Hamiltonian flow}

In this section, we study 'rotating loops' in the Hamiltonian flow, introduced in Definition 8.3 and their connection to the emergence of points of non-differentiability. We give a short overview:

- Under the Condition of Theorem 3.6 (b), we find a rotating loop in the Hamiltonian flow that intersects $\mathscr{G}$ : solutions of the flow on this loop come back to the same point in some finite time. (Lemma 8.6.)

- Consider some fixed rotating loop. A homotopy argument, considering the punctured space $\mathbb{K} \times \mathbb{R}$ without the interior of this loop, can be used to show that any intersection of the loop with the graph $\mathscr{G}$ of the gradient of a rate function $I_{0}$ implies that for large $t$ there is an element of $\mathscr{G}_{t}$ 'above' the loop. Similarly, one can show that for large $t$ there is an element of $\mathscr{G}_{t}$ 'below' the loop. This will lead to the creation of an overhang. (Theorem 8.5.)

- First, we characterise rotating loops by their insides. (Lemma 8.4)

Definition 8.3. We call a set $\mathfrak{L} \subset \mathbb{K} \times \mathbb{R}$ a loop if there exist $a, b \in \mathbb{K}, a<b$ and functions $g, h \in C[a, b]$ with $g(x)<h(x)$ for $x \in(a, b), g(a)=h(a)$ and $g(b)=h(b)$ such that $\mathfrak{L}=g[a, b] \cup h[a, b]$.

Let $(x, p) \in \mathbb{K} \times \mathbb{R}$ be a non-stationary point, $E=H(x, p)$. If there exists a $t_{0}>0$ such that $\left(X_{t_{0}}^{x, p}, P_{t_{0}}^{x, p}\right)=(x, p)$, then $\mathfrak{L}=\left\{\left(X_{t}^{x, p}, P_{t}^{x, p}\right): t \in\left[0, t_{0}\right]\right\}$ is called a rotating loop. $E$ is called the energy of $\mathfrak{L}$. Let $a$ be the largest value and $b$ be the smallest value for which $\mathfrak{L} \subseteq[a, b] \times \mathbb{R}$. The set $A=\{(x, p) \in[a, b] \times \mathbb{R}: H(x, p)<E\}$ is called the inside of $\mathfrak{L}$.

Note that if $H$ satisfies Assumption 2.10, then a rotating loop is indeed a loop. 


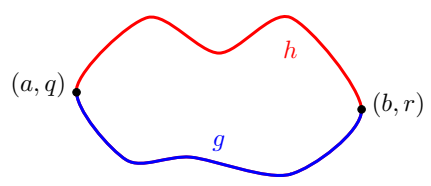

(a)

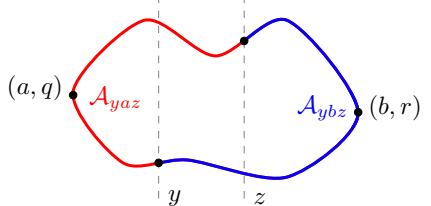

(b)

Figure 3: (a) Functions $g$ and $h$, (b) The $\operatorname{arcs} \mathscr{A}_{y, a, z}$ and $\mathscr{A}_{y, b, z}$.

\section{Lemma 8.4. Let $H$ satisfy Assumption 2.10.}

Let $E \in \mathbb{R}$. Suppose that $A$ is nonempty, relatively compact and a connected component of $H^{-1}(-\infty, E)$ and that $\partial A$ is a connected component of $H^{-1}(\{E\})$. Then $\partial A$ is a loop and with $a, b, g, h$ as in Definition 8.3, the functions $g$ and $h$ are $C^{1}$ on $(a, b)$. We write $q:=g(a)=h(a)$ and $r:=g(b)=h(b)$.

Then $\partial_{p} H(a, q)=\partial_{p} H(b, r)=0$ and

$$
\begin{aligned}
& \partial_{p} H(x, h(x))>0, \quad \partial_{p} H(x, g(x))<0 \quad \text { for } x \in(a, b), \\
& \partial_{x} H(a, q) \neq 0 \quad \Longrightarrow \quad \partial_{x} H(a, q)<0, \\
& \partial_{x} H(b, r) \neq 0 \quad \Longrightarrow \quad \partial_{x} H(b, r)>0 .
\end{aligned}
$$

$\mathfrak{L}$ is a rotating loop $i$ f $^{6}$ the inside of $\mathfrak{L}$ is a set $A$ as above with

$$
\partial_{x} H(a, q) \neq 0 \quad \text { and } \quad \partial_{x} H(b, r) \neq 0 .
$$

Proof. Let $a$ and $b$ be the smallest and largest element in the set $\{x: \exists p,(x, p) \in \bar{A}\}$, respectively. By strict convexity of $p \mapsto H(x, p)$, we have $\partial A=\{(x, p) \in \bar{A}: H(x, p)=$ $E\}$. This strict convexity, together with the implicit function theorem, implies the existence of $g, h$ as in the first part of Definition 8.3. which are $C^{1}$ on $(a, b)$ and it implies 62 .

We prove (63), the proof of $(64)$ being similar. Suppose that $\partial_{x} H(a, q) \neq 0$. By the implicit function theorem, $\partial A$ can be described by a $C^{1}$ function around $(a, q)$, i.e., there exists a $\delta>0$ and a $C^{1}$ function $j:(q-\delta, q+\delta) \rightarrow[-1,1]$ such that $\{(j(p), p): p \in(q-\delta, q+\delta)\}=\{(x, p) \in \partial A: p \in(q-\delta, q+\delta)\}$ and

$$
j^{\prime}(p)=-\frac{\partial_{p} H(j(p), p)}{\partial_{x} H(j(p), p)} \quad(p \in(q-\delta, q+\delta)) .
$$

Note that $j^{\prime}(p)=0$ if and only if $p=q$.

Therefore for $p \in(q, q+\delta)$ we have $j^{\prime}(q)>0$ and thus by (66) and 62$) \partial_{x} H(j(p), p)<$ 0 . Then $\partial_{x} H(a, q)<0$ follows by taking a limit.

We will now show that $\partial_{x} H(a, q) \neq 0$ implies that one has a rotation around $a$ in the following sense. Let $y, z \in(a, b)$ and $\mathscr{A}_{y, a, z}$ be the arc from $y$ to $z$ via $a$ (see Figure $3(b)$ ),

\footnotetext{
${ }^{6}$ Actually "if and only if"; as we don't use this we leave out the proof.
} 
i.e., $\mathscr{A}_{y, a, z}:=\{(x, g(x)): x \in(a, y)\} \cup\{(a, q)\} \cup\{(x, h(x)): x \in(a, z)\}$. We show that every point in the arc passes thought the point $(z, h(z))$ in a finite and bounded time: We show that there exists a $t_{a}$ such that for all $(x, p) \in \mathscr{A}_{y, a, z}$ there exists a $t_{0}<t_{a}$ such that

$$
\left(X_{t}^{x, p}, P_{t}^{x, p}\right) \in \mathscr{A}_{y, a, z} \text { for all } t<t_{0}, \quad\left(X_{t_{0}}^{x, p}, P_{t_{0}}^{x, p}\right)=(z, h(z)) .
$$

Let $q_{1}, q_{2} \in(q-\delta, q+\delta)$ be such that $q_{1}<q<q_{2}$. We may assume that $x_{1}:=j\left(q_{1}\right)<y$ and $x_{2}:=j\left(q_{2}\right)<z$. There exists an $\epsilon>0$ such that

$$
\begin{array}{cc}
\partial_{p} H(x, g(x))<\epsilon & \left(x \in\left[x_{1}, y\right]\right), \\
-\partial_{x} H(j(p), p)>\epsilon & \left(p \in\left[q_{1}, q_{2}\right]\right), \\
\partial_{p} H(x, h(x))>\epsilon & \left(x \in\left[x_{2}, z\right]\right) .
\end{array}
$$

Therefore there exist $t_{1}, t_{2}, t_{3}$ such that for all $x \in\left[x_{1}, y\right]$ there exists a $t_{0} \leq t_{1}$ such that $X_{t}^{x, g(x)}>x_{1}$ for $t<t_{0}, X_{t_{0}}^{x, g(x)}=x_{1}$; for all $x \in\left[x_{2}, z\right]$ there exists a $t_{0} \leq t_{2}$ such that $X_{t}^{x, h(x)}<z$ for $t<t_{0}, X_{t_{0}}^{x, h(x)}=z$; and for all $p \in\left[q_{1}, q_{2}\right]$ there exists a $t_{0} \leq t_{3}$ such that $P_{t}^{j(p), p}<q_{2}$ for $t<t_{0}, P_{t_{0}}^{j(p), p}=q_{2}$. Now by taking $t_{a}=t_{1}+t_{2}+t_{3}$, we leave it to the reader to check that the claim follows.

Similarly, one shows that $\partial_{x} H(b, r) \neq 0$ implies rotation around $b$ (along $\operatorname{arcs} \mathscr{A}_{y, b, z}$, see Figure $3(\mathrm{~b}))$. Therefore it follows that $\mathfrak{L}=\partial A$ is a rotating loop if $\partial_{x} H(a, q) \neq 0$ and $\partial_{x} H(b, r) \neq 0$.

Theorem 8.5. Let $H$ satisfy Assumption 2.10. Suppose that $\mathfrak{L}$ is a loop, $\mathscr{G} \cap \mathfrak{L} \neq \emptyset$, and that $\left(x_{1}, p_{1}\right)$ and $\left(x_{2}, p_{2}\right)$ with $x_{1} \leq x_{2}$ are those points in $\mathscr{G} \cap \mathfrak{L}$ such that $\{(x, p) \in$ $\left.\mathscr{G}: x<x_{1}\right\} \cap \mathfrak{L}=\emptyset$ and $\left\{(x, p) \in \mathscr{G}: x>x_{2}\right\} \cap \mathfrak{L}=\emptyset$. Let $x_{0} \in\left[x_{1}, x_{2}\right]$.

(a) If $t_{1} \geq 0$ is such that $\left(X_{t_{1}}^{x_{1}, I_{0}^{\prime}\left(x_{1}\right)}, P_{t_{1}}^{x_{1}, I_{0}^{\prime}\left(x_{1}\right)}\right)=\left(x_{0}, h\left(x_{0}\right)\right)$, then for all $t \geq t_{1} \mathscr{G}_{t}$ intersects the half-line $\left\{x_{0}\right\} \times\left[h\left(x_{0}\right), \infty\right)$.

(b) If $t_{2} \geq 0$ is such that $\left(X_{t_{2}}^{x_{2}, I_{0}^{\prime}\left(x_{2}\right)}, P_{t_{2}}^{x_{2}, I_{0}^{\prime}\left(x_{2}\right)}\right)=\left(x_{0}, g\left(x_{0}\right)\right)$, then for all $t \geq t_{2} \mathscr{G}_{t}$ intersects the half-line $\left\{x_{0}\right\} \times\left(-\infty, g\left(x_{0}\right)\right]$.

Consequently, if $\mathfrak{L}$ is a rotating loop, then there exists a $t_{0}$ such that $\mathscr{G}_{t}$ contains an overhang for all $t \geq t_{0}$.

Proof. We prove (a) only, as the proof of (b) is similar. Suppose that $t_{1}$ is as in (a). Write $\mathrm{L}$ for the half-line $\left\{x_{0}\right\} \times\left[h\left(x_{0}\right), \infty\right)$ and write $\Theta:[0, \infty) \times\left[\partial_{-}, \partial_{+}\right] \rightarrow \mathfrak{S}$ for the function given by $\Theta(t, x)=\Psi\left(t, x, I_{0}^{\prime}(x)\right.$ ) (with the convention that $I_{0}^{\prime}$ is defined in $\partial_{-}$ and $\partial_{+}$as $-\infty$ and $\infty$, respectively) where $\Psi$ is as in Proposition 6.1. Note that $\Theta$ is continuous by continuity of $\Psi$ and because $I_{0} \in C^{1, \partial}(\mathbb{K})$. We prove that for all $t \geq t_{1}$ there exists an $x \in\left(\partial_{-}, x_{1}\right]$ such that $\Theta(t, x) \in L$.

The idea is that the curve $\Theta\left(t,\left[\partial_{-}, x_{1}\right]\right)$ is pulled through the line $L$ by the rotation of $\Phi_{t}\left(x_{1}\right)$ over $\mathfrak{L}$, and as it is connected to $\left(\partial_{-},-\infty\right)$ it will be connected via $L$ for all larger times. We use an argument using homotopy theory to prove this. 
Let $\sigma:[0,1] \rightarrow\left[\partial_{-}, x_{1}\right]$ be a continuous function with $\sigma(0)=\partial_{-}$and $\sigma(1)=x_{1}$. Define the map $\Gamma:[0, \infty) \times[0,3]$ as follows, $\Gamma(t,[0,1])$ is the set $\Theta\left(0,\left[\partial_{-}, x_{1}\right]\right) ; \Gamma(t,[1,2])$ is the set $\Theta\left([0, t], x_{1}\right)$, i.e., the Hamiltonian path starting at $\left(x_{1}, I_{0}^{\prime}\left(x_{1}\right)\right)$ up to time $t$; $\Gamma(t,[2,3))$ is the set $\Theta\left(t,\left[\partial_{-}, x_{1}\right]\right)$ (see also Figure 4); more precisely, for $t \in[0, \infty)$ and $s \in[0,1]$

$$
\begin{aligned}
\Gamma(t, s) & :=\Theta(0, \sigma(s)), \\
\Gamma(t, 1+s) & :=\Theta\left(t s, x_{1}\right), \\
\Gamma(t, 2+s) & :=\Theta(t, \sigma(1-s)) .
\end{aligned}
$$

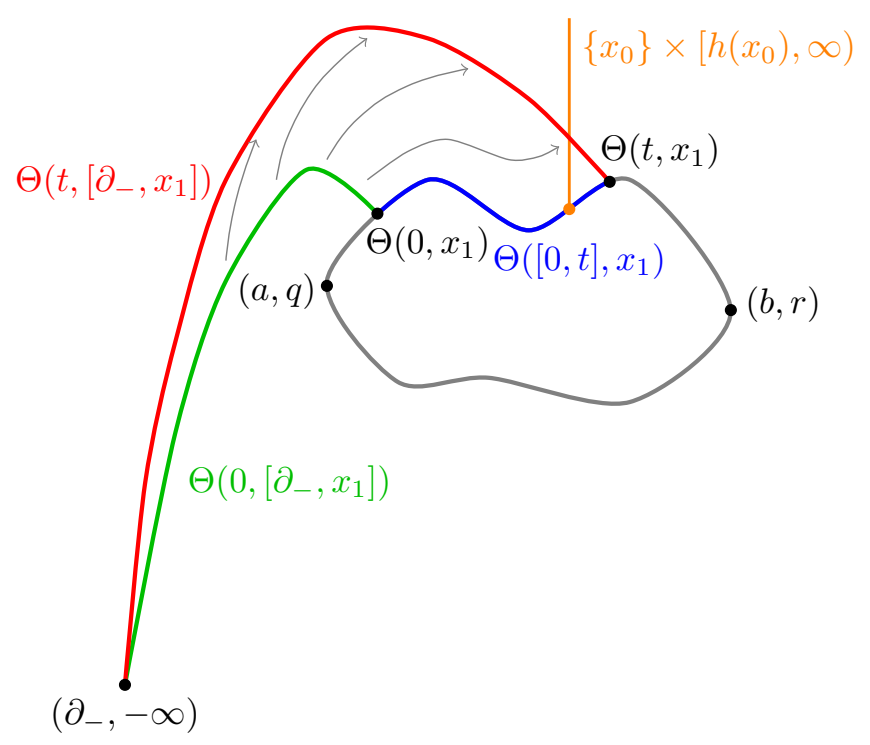

Figure 4: $\Theta\left(0,\left[\partial_{-}, x_{1}\right]\right), \Theta\left(t,\left[\partial_{-}, x_{1}\right]\right)$ and $\Theta\left([0, t], x_{1}\right)$.

$\Gamma$ is continuous and therefore a path homotopy between the paths $\gamma_{t}:[0,3] \rightarrow$ $\mathfrak{S} \backslash A$ for $t \in[0, \infty)$ where $\gamma_{t}(s)=\Gamma(t, s)$ and $A$ is the inside of $\mathfrak{L}$. Hence $\gamma_{t}$ is pathhomotopic to $\gamma_{0}$ in the space $\mathfrak{S} \backslash A$ and thus to the single point $\left(\partial_{-}, \infty\right)$. That a closed path is homotopic to another one in a topological space, basically means that one can continuously transform one path in that space to the other one, being homotopic to a point means homotopic to a path that stays at that point. In [Run05, Section 5] one finds the necessary background for homotopy theory.

We will use the following fact: A closed path $\gamma:[0,3] \rightarrow \mathfrak{S} \backslash\left\{\left(x_{0}, p_{0}\right)\right\}$ is homotopic to the point $\left(\partial_{-},-\infty\right)$ if and only if there exist $s_{1}, s_{2} \in[0,3], s_{1}<s_{2}$ such that $\gamma\left(s_{1}\right), \gamma\left(s_{2}\right)$ are either both in $\left\{x_{0}\right\} \times\left(p_{0}, \infty\right)$ or both in $\left\{x_{0}\right\} \times\left(-\infty, p_{0}\right)$ and $s_{1}, s_{2}$ are the first and last time such that the path crosses the line $\left\{x_{0}\right\} \times \mathbb{R}$, respectively, i.e.,

$$
\gamma\left(\left[0, s_{1}\right)\right) \cup \gamma\left(\left(s_{2}, 3\right]\right) \subseteq\left(-\infty, x_{0}\right) \times \mathbb{R} \cup\left\{\left(\partial_{-},-\infty\right)\right\} .
$$


This fact can be proven as follows; for simplicity with $(0,0)$ instead of $\left(x_{0}, p_{0}\right)$ and $(-1,0)$ instead of $\left(\partial_{-},-\infty\right)$. Every closed path is homotopic to $s \mapsto(-\cos (2 \pi k s), \sin (2 \pi k s))$ for some $k \in \mathbb{Z}$ (see, e.g. [Run05, Example 5.2.7]). It is straightforward to check that such $s_{1}$ and $s_{2}$ do not exist in case $k \neq 0$.

Let $p_{0} \in\left(g\left(x_{0}\right), h\left(x_{0}\right)\right)$. Let $t \geq t_{1}$. As $x_{1}<x_{0}, s_{1} \geq 1$. By the choice of $t_{1}$ we have $s_{1} \in[1,2]$ and $\gamma_{t}\left(s_{1}\right) \in\left\{x_{0}\right\} \times\left(p_{0}, \infty\right)$. As the $\partial_{p} H$ at $\left(x_{0}, h\left(x_{0}\right)\right)$ is strictly positive (see $(62)$ ), the $s_{2}$ as above cannot be in $[1,2)$. Thus $s_{2} \in[2,3]$, which proves that there exists an $x \in\left[\partial_{-}, x_{1}\right]$ such that $\Theta(t, x) \in\left\{x_{0}\right\} \times\left[h\left(x_{0}\right), \infty\right)$.

The following lemma establishes the existence of a rotating loop $\mathfrak{L}$ with $\mathscr{G} \cap \mathfrak{L} \neq \emptyset$ under the assumptions made in Theorem 3.6(b), so that with Theorem 8.5 the statement of Theorem 3.6)(b) is proven.

Lemma 8.6. Assume Assumption 2.10 and suppose that $H$ is $C^{3}$. Suppose that $m_{1}, m_{2} \in$ $\mathbb{K}^{\circ}$ are two points such that $m_{1}<m_{2}$ and

(i) $\partial_{p} H\left(m_{1}, 0\right)=0=\partial_{p} H\left(m_{2}, 0\right)$ and $\partial_{p} H(x, 0) \neq 0$ for all $x \in\left(m_{1}, m_{2}\right)$,

(ii) $\partial_{x} \partial_{p} H\left(m_{1}, 0\right) \neq 0$ and $\partial_{x} \partial_{p} H\left(m_{2}, 0\right) \neq 0$.

If $\left\{(x, p) \in\left(m_{1}, m_{2}\right) \times \mathbb{R} \mid H(x, p)<0\right\} \cap \mathscr{G} \neq \emptyset$, then there exists a rotating loop $\mathfrak{L}$ such that $\mathscr{G} \cap \mathfrak{L} \neq \emptyset$.

Proof. By Lemma 8.4 it is sufficient to show that there exists an $E<0$ such that the set

$$
A=\left\{(x, p) \in\left(m_{1}, m_{2}\right) \times \mathbb{R} \mid H(x, p)<E\right\},
$$

is as in Lemma 8.4 such that $\mathscr{G} \cap A \neq \emptyset$ and $(65)$ holds.

To find such an $E$ so that $A$ is connected, we consider the function that gives the minimum of $H$ at $x, \mathbf{E}(x)=\inf _{p \in \mathbb{R}} H(x, p)$. We use that a set $A$ as defined in (67) is connected as soon as $\left(x_{1}, p_{1}\right),\left(x_{2}, p_{2}\right) \in A$ imply that $\mathbf{E}(x)<E$ for all $x \in\left[x_{1}, x_{2}\right]$. Let $p(x)=\operatorname{argmin}_{q \in \mathbb{R}} H(x, q)$, so that $q=p(x)$ if and only if $\partial_{p} H(x, q)=0$ and $\mathbf{E}(x)=$ $H(x, p(x))$. By Assumption (i) $\mathbf{E}\left(m_{1}\right)=0=\mathbf{E}\left(m_{2}\right)$ and $\mathbf{E}<0$ on $\left(m_{1}, m_{2}\right)$. As $H$ is $C^{3}, p$ is $C^{2}$ by the implicit function theorem and

$$
p^{\prime}(x)=-\frac{\partial_{x} \partial_{p} H(x, p(x))}{\partial_{p}^{2} H(x, p(x))} .
$$

Note that $\mathbf{E}$ is also $C^{2}$. We show that $\mathbf{E}^{\prime \prime}\left(m_{*}\right)<0$ for $m_{*} \in\left\{m_{1}, m_{2}\right\}$. We have

$$
\mathbf{E}^{\prime}(x)=\partial_{x}[H(x, p(x))]=\partial_{x} H(x, p(x))+\partial_{p} H(x, p(x)) p^{\prime}(x)=\partial_{x} H(x, p(x)),
$$

as $\partial_{p} H(x, p(x))=0$. Moreover,

$$
\mathbf{E}^{\prime \prime}(x)=\partial_{x}^{2} H(x, p(x))+\partial_{x} \partial_{p} H(x, p(x)) p^{\prime}(x) .
$$

As for $m_{*} \in\left\{m_{1}, m_{2}\right\}, p\left(m_{*}\right)=0$ we have $\partial_{x}^{2} H\left(m_{*}, p\left(m_{*}\right)\right)=0($ as $H(x, 0)$ for all $x)$, Hence by (68) and Assumption (ii)

$$
\mathbf{E}^{\prime \prime}\left(m_{*}\right)=-\frac{\left[\partial_{x} \partial_{p} H\left(m_{*}, 0\right)\right]^{2}}{\partial_{p}^{2} H\left(m_{*}, 0\right)}<0 .
$$


Let $\delta>0$ be such that $\mathbf{E}^{\prime \prime}<0$ on $\left[m_{1}, m_{1}+\delta\right]$ and $\left[m_{2}-\delta, m_{2}\right]$ and thus

$$
\mathbf{E}^{\prime}<0 \text { on }\left[m_{1}, m_{1}+\delta\right] \text { and } \mathbf{E}^{\prime}>0 \text { on }\left[m_{2}-\delta, m_{2}\right] .
$$

Let $\epsilon$ be such that $-\epsilon$ is the maximum of $\mathbf{E}$ on $\left[m_{1}+\delta, m_{2}-\delta\right]$. Then for all $E \in(0, \epsilon)$ the set (67) is nonempty, connected and its closure is a compact set and a connected component of $H^{-1}(-\infty, E]$. Moreover, (65) is satisfied by (69) and (70). By choosing $E$ small enough, one obtains $\mathscr{G} \cap A \neq \emptyset$.

Remark 8.7. Suppose that $A$ is a set as in Lemma 8.4 with $\mathscr{G} \cap A \neq \emptyset$ and $\partial_{x} H(a, q) \neq 0$ and $\partial_{x} H(b, r)=0$ (or the other way around). Then one can also conclude the creation of overhangs for large times as follows. There exists a point $\left(x_{0}, p_{0}\right) \in \mathscr{G} \cap A$ with a lower energy, i.e., $H\left(x_{0}, p_{0}\right)<E$. Therefore $X_{t}^{x_{0}, p_{0}}$ stays bounded away from $b$, while by Lemma 8.4 (see the proof where we show the rotation of the $\left.\operatorname{arcs} \mathscr{A}_{y, a, z}\right) X_{t}^{x_{i}, I_{0}^{\prime}\left(x_{i}\right)}$ for large $t$ will be arbitrarily close to $b$, where $\left(x_{1}, I_{0}^{\prime}\left(x_{1}\right)\right.$ and $\left(x_{2}, I_{0}^{\prime}\left(x_{2}\right)\right.$ are distinct elements of $\mathscr{G} \cap \partial A$.

\section{Proof of Theorem 3.10 and Theorem 3.11}

We end our paper with the application of the developed methods for two main classes of examples. To be precise, we prove Theorem 3.10 . The proof will only be carried out for the \pm 1 -space-model as the proof of the $\mathbb{R}$-space-model is up to a change of notation the same.

Proof Theorem 3.10 for the \pm 1 -space-model. (a) is immediate as $H\left(x, I_{0}^{\prime}(x)\right)=0$, and hence $\mathscr{G}_{t}=\mathscr{G}_{0}$.

For the proof of (b) we apply Theorem 3.5 (b) $I_{0}$ is strictly convex at infinity (see e.g. (38) and $H$ preserves order at infinity by Proposition 3.9)(a).

For the proof of (c) we apply Theorem 3.5 (a). For $\alpha \leq 1, I_{0}^{\prime}$ is strictly increasing (see also (37) and (38)). For $\beta \in[0,1]$ by Proposition 3.9(a) $H$ preserves order on $\mathbb{7}_{0,0}^{\circ} \cup\{(0,0)\} \cup \mathfrak{K}_{0,0}^{\circ}$, of which the graph of $I_{0}^{\prime}, \mathscr{G}$, is a subset.

For the proof of $(\mathrm{d})$ we apply Theorem 3.6(a). We consider the stationary point $x_{0}=0$. First, note that $m=\partial_{x} \partial_{p} H(0,0)=2(\beta-1) \neq 0$ and $c=\partial_{p}^{2} H(0,0)=4$. As the Hamiltonian is $C^{\infty}$, there is a $C^{1}$ linearization of the Hamiltonian flow at $(0,0)$ by Theorem 8.1. Explicit calculation yields $I_{0}^{\prime}(0)=0$ and $I_{0}^{\prime \prime}(0)=(1-\alpha)$. The condition $I_{0}^{\prime \prime}(0)<-\frac{2 m}{c} \wedge 0$ translates into $1 \vee \beta<\alpha$ and (27) into $t_{1}$ as in (ii).

For (e) first we make the following observations. By Remark 3.8 we see that $H(x, p)=0$ if and only if $p=0$ or $p=f_{\beta}(x)$, where

$$
f_{\beta}(x)=\operatorname{arctanh}(x)-\beta x .
$$

As $\beta>1$ and $\operatorname{arctanh}^{\prime}(x)=\frac{1}{1-x^{2}}$, the function $f_{\beta}$ intersects the $x$-axis at 3 points, $m_{-}, 0, m_{+}$. As $1<\alpha<\beta$, then function $I_{0}^{\prime}$, being $f_{\alpha}$ (see (37), $f_{\alpha}$ is as $f_{\beta}$ in (71)), 


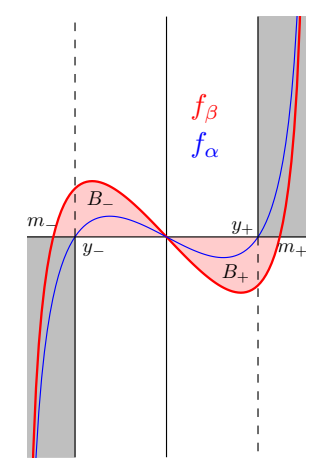

Figure 5: $f_{\beta}, f_{\alpha}$, their zero's and regions $B_{ \pm}$.

intersects the $x$-axis at 3 points too, $y_{-}, 0, y_{+}$and $m_{-}<y_{-}<0<y_{+}<m_{+}$(see Figure 5). Moreover, the graph of $I_{0}^{\prime}$ has a nonempty intersection with $B_{-}$and $B_{+}$, where

$$
\begin{aligned}
& B_{-}=\left\{(x, p) \in\left(m_{-}, 0\right) \times \mathbb{R}: H(x, p)<0\right\}, \\
& B_{+}=\left\{(x, p) \in\left(0, m_{+}\right) \times \mathbb{R}: H(x, p)<0\right\} .
\end{aligned}
$$

First we will show that $\mathscr{G}_{t}$ has no overhang at $\gamma_{t}\left(y_{-}\right)$, at 0 and at $\gamma_{t}\left(y_{+}\right)$. Let $x<y_{-}$, then $I_{0}^{\prime}(x)<0$ and $H\left(x, I_{0}^{\prime}(x)\right) \neq 0$, so that $P_{t}^{x, I_{0}^{\prime}(x)}<0$ for all $t \geq 0$. With Lemma 7.1 this implies that $\gamma_{t}(x)<\gamma_{t}\left(y_{-}\right)$for all $t$. On the other hand, $\Phi_{t}(x) \in B_{1}$ for all $x \in\left(y_{-}, 0\right)$ and all $t$, whence Lemma 7.1 implies $\gamma_{t}\left(y_{-}\right)<\gamma_{t}(x)$. So that (by symmetry) we obtain for $x_{1}, x_{2}, x_{3}, x_{4}$ with $x_{1}<y_{-}<x_{2}<0<x_{3}<y_{+}<x_{4}$ and all $t \geq 0$ (as long as $\gamma_{t}\left(x_{i}\right)$ exists)

$$
\gamma_{t}\left(x_{1}\right)<\gamma_{t}\left(y_{-}\right)<\gamma_{t}\left(x_{2}\right)<0<\gamma_{t}\left(x_{3}\right)<\gamma_{t}\left(y_{+}\right)<\gamma_{t}\left(x_{4}\right) .
$$

So indeed, $\mathscr{G}_{t}$ has no overhangs at $\gamma_{t}\left(y_{-}\right), 0$ and $\gamma_{t}\left(y_{+}\right)$.

By Proposition 2.16 we obtain that $I_{t}$ is non-differentiable at two points $x_{-} \in\left(m_{-}, 0\right)$ and $x_{+} \in\left(0, m_{+}\right)$, as soon as $\mathscr{G}_{t}$ has an overhang in $B_{-}$and $B_{+}$. The existence of a $t_{2}$ such that for $t \geq t_{2}$ this is the case follows from Theorem 3.6|(b), as soon as the conditions (i) and (ii) are satisfied for both $\left(m_{1}, m_{2}\right)=\left(m_{-}, 0\right)$ and $\left(m_{1}, m_{2}\right)=\left(0, m_{+}\right)$. We show that this is indeed the case.

By (32) we see that $\partial_{p} H(x, 0)=0$ if and only if $\operatorname{arctanh}(x)=\beta x$, whence it is clear that (i) of Theorem 3.6(b) is satisfied. By (34) we see that $\partial_{p} \partial_{x} H(x, 0)=0$ if and only if

$$
1-\beta+\beta x \tanh (\beta x)=0 .
$$

$f_{\beta}$ has a local maximum or minimum at $x$ if $f_{\beta}^{\prime}(x)=0$, which is the case if and only if $1-\beta+\beta x^{2}=0$. At those points $f_{\beta}$ is not equal to zero. By definition of $m_{ \pm}$we have $1-\beta+\beta m_{ \pm} \tanh \left(\beta m_{ \pm}\right)=1-\beta+\beta m_{ \pm}^{2}$, from which we conclude that $(72)$ does not hold for $x=m_{ \pm}$. Similarly, we have that $(\sqrt{72})$ does not hold at $x=0$. This proves condition (ii). 
Proof of Theorem 3.11. First we make the following observation.

Observation Note that the Hamiltonian dynamics for the momentum is autonomous: $\dot{P}=\sinh (2 P)$. Therefore $t_{x_{1}, p}=t_{x_{2}, p}<\infty$ for all $p \neq 0$ and $x_{1}, x_{2} \in[-1,1]$, and, if $0<\left|p_{1}\right|<\left|p_{2}\right|$, then $t_{x_{1}, p_{1}}<t_{x_{2}, p_{2}}$. By Proposition $3.9 H$ preserves order $[-1,1] \times \mathbb{R}$.

(a) Let $x_{0}$ be the unique solution to $I_{0}^{\prime}=0$. Then there exists a $\delta>0$ and a neighbourhood $U$ of $x_{0}$, such that $I_{0}^{\prime}$ is strictly increasing on $U$ and such that $I_{0}^{\prime}(x)<\delta$ implies $x \in U$. Let $t^{*}=t_{0, \delta}$. As we saw in our observation this implies that $t_{z, I_{0}^{\prime}(z)}<t^{*}$ for $z \notin U$ and thus $E_{t}$ as in Proposition 6.2 is a subset of $U$ for $t \geq t^{*}$. As $H$ preserves order, this implies that $\gamma_{t}$ is strictly increasing on $E_{t}$, i.e., $\mathscr{G}_{t}$ is a graph.

(b) Fix $\alpha>1$. We write

$$
g_{\theta}(x)=\operatorname{arctanh}(x)-\alpha x-\theta .
$$

There exists a $z>0$, such that $-z$ is a local maximum and $z$ is a local minimum of $g_{\theta}$ for all $\theta$. Let $\kappa=g_{0}(-z)=-g_{0}(z)$ (note that the graph of $g_{\kappa}$ looks like the graph of $I_{0}^{\prime}$, the dashed blue graph - - - - in Figure 3.2). For the rest of the proof we let $\theta$ be fixed and such that $-\theta>\kappa>0$. (By symmetry we could have also treated $\theta>\kappa$.)

Let $t_{1}=t_{-z, g_{\theta}(-z)}$. We show in STEP 1 that there exists an overhang at a time before $t_{1}$, so that we can take $t_{0}$ to be be the infimum of all times at which there is an overhang. By the choice of $\theta, g_{\theta}=I_{0}^{\prime}$ has a unique zero, which is the unique global minimiser of $I_{0}$. By (a) there exists a $t^{*}$ such that $I_{t}$ is $C^{1}$ and so that there is no overhang for $t \geq t^{*}$. We let $t_{2}$ be the supremum of all times at which there is an overhang. In STEP 2 we show that $I_{t}$ is non-differentiable for $t \in\left(t_{0}, t_{1}\right)$.

$\underline{\text { STEP } 1}$ By our observation, $t_{z, I_{0}^{\prime}(z)}<t_{1}$ and thus $M:=\lim _{t \uparrow t_{1}} P_{t}^{z, g_{\theta}(z)}<\infty$. By Lemma 4.4 there exists a $u \in(0,1)$ such that $\gamma_{t}(z) \leq u$ for all $t \leq t_{1}$. As $P_{t}^{-z, g_{\theta}(-z)} \rightarrow \infty$ as $t \uparrow t_{1}$, by Lemma 4.4 again (or Lemma 4.6) we have $\lim _{t \uparrow t_{1}} \gamma_{t}(-z)=1$. Hence there exists a $t<t_{1}$ such that $\gamma_{t}(-z)>u \geq \gamma_{t}(z)$, i.e., there is an overhang.

STEP 2 Let $y<-z$ be the unique point such that $I_{0}^{\prime}(y)=0$, and let $w>z$ be such that $g_{\theta}(w)=g_{\theta}(-z)$. We show that for all $t<t_{1}$, there is no overhang in $\left(-1, \gamma_{t}(y)\right)$ and in $\left(\gamma_{t}(w), 1\right)$, so that Proposition 2.16 implies that $I_{t}$ is non-differentiable for $t \in\left(t_{0}, t_{1}\right)$. But this follows as $g_{\theta}$ is strictly increasing on $(-1,-z)$ and on $(z, 1)$, and $g_{\theta}<0$ on $(-1, y)$ and $g_{\theta}>g_{\theta}(-z)$ on $(w, 1)$ by the fact that $H$ preserves order.

Remark 9.1. Note that in our proof $t_{1}$ is strictly less than $t_{2}$; Indeed, for $t>t_{1}$ and $t<t_{z, g_{\theta}(z)}$ the set $\Phi_{t}\left(E_{t} \cap[-1,-z]\right)$ connects $(-1,-\infty)$ with $(1, \infty)$ and the set $\Phi_{t}\left(E_{t} \cap(-z, 1]\right)$ is non-empty as it contains $\Phi_{t}(z)$.

\section{A The verification of Assumption 4.1 for the main exam- ples}

In this appendix, we show that Hamiltonians that satisfy Assumption 2.10 (a) in fact satisfy Assumption 2.10 (b). 


\section{A.1 Verification for the Curie-Weiss example}

Lemma A.1. Let $H$ satisfy Assumption 2.10(a) for the \pm 1 -space-model, then $H$ satisfies Assumption 4.1 .

For convenience in the proof, we recall part of [CS04, Corollary A.2.7].

Lemma A.2. $H$ is as in (15) then for all $x \in(-1,1)$ and $v \in \mathbb{R}$ there exists a unique $p$ for which $v=\partial_{p} H(x, p), \mathscr{L}(x, v)=p \partial_{p} H(x, p)-H(x, p), \partial_{x} \mathscr{L}(x, v)=-\partial_{x} H(x, p)$, and $\partial_{v} \mathscr{L}(x, v)=p$.

Proof of Lemma A.1. (a) follows from Assumption 2.3(b). (c) follows directly from Assumption 2.3(a). For (d) note that $p(x):=\operatorname{argmin}_{p} H(x, p)$ satisfies $\partial_{p} H(x, p)=0$. By Assumption 2.3 (a), we find $\partial_{p} H(-1, p)>0$ and $\partial_{p} H(1, p)<0$ for all $p$, implying by the continuity of $\partial_{p} H$ that (d) holds.

In addition, (e) follows by an explicit computation using Assumption 2.3. (c).

We are left to verify (b). Pick some some compact set $K \subseteq(-1,1)$.

We consider the function $\theta_{K}=\theta$ with $\theta(r)=\frac{1}{4} \max \{r \log r, 1\}$. Property (i) is immediate.

We proceed with the proof of (ii). Let $M \geq 0$. Note that

$$
\sup \{\theta(r+m): r \in[0, M \vee 2], m \in[0, M]\}<\infty .
$$

Hence it is sufficient for (ii) to show that $\theta(r+m) / \theta(r)$ is bounded from above for $r \geq M \vee 2$ and $m \in[0, M]$. For such $m$ and $r$ we have $m \leq M \leq r$ and thus

$$
\frac{\theta(r+m)}{\theta(r)}=\frac{r+m}{r} \frac{\log (r+m)}{\log r} \leq 2 \frac{\log (r+m)}{\log r} .
$$

The latter ratio is indeed bounded.

For (iii), by Lemma A.2 it suffices to show the existence of a constant $c$ such that

$$
p \partial_{p} H(x, p)-H(x, p) \geq \theta\left(\left|\partial_{p} H(x, p)\right|\right)-c \quad \text { for all } x \in K, p \in \mathbb{R} .
$$

For (iv), by Lemma A.2 it suffices to show the existence of a constant $c$ such that

$$
\left|\partial_{x} H(x, p)\right|+|p| \leq c \theta\left(\left|\partial_{p} H(x, p)\right|\right) \quad \text { for all } x \in K, p \in \mathbb{R} .
$$

We will consider the following computations and estimations

$$
\begin{aligned}
& \partial_{p} H(x, p)=v_{+}(x) 2 e^{2 p}-v_{-}(x) 2 e^{-2 p}, \\
& \partial_{x} H(x, p)=v_{+}^{\prime}(x)\left[e^{2 p}-1\right]+v_{-}^{\prime}(x)\left[e^{-2 p}-1\right],
\end{aligned}
$$

By Assumption 2.3(a) there exists $0<a<b$ such that

$$
v_{-}(x), v_{+}(x) \in[a, b] \quad \text { and } \quad\left|v_{-}^{\prime}(x)\right|,\left|v_{+}^{\prime}(x)\right| \leq b \quad \text { for all } x \in K,
$$


Then

$$
\left|\partial_{x} H(x, p)\right| \leq \begin{cases}b\left(e^{2 p}-1\right)+b=b e^{2 p} & \text { if } p \geq 0, \\ b+b\left(e^{-2 p}-1\right)=b e^{-2 p} & \text { if } p \leq 0 .\end{cases}
$$

Set $\psi(p)=2 p e^{2 p}-e^{2 p}+1$. Then $\psi \geq 0$, and

$$
\begin{aligned}
p \partial_{p} H(x, p)-H(x, p) & =v_{+}(x) \psi(p)+v_{-}(x) \psi(-p) \\
& \geq \begin{cases}\psi(p) v_{+}(x) & \text { if } p \geq 0 \\
\psi(-p) v_{-}(x) & \text { if } p \leq 0 .\end{cases}
\end{aligned}
$$

Let $p_{0}>0$ be such that

$$
a e^{2 p} \geq 2, \quad a e^{2 p} \geq b e^{-2 p}+\frac{a}{2} e^{2 p} \quad \text { for } p \geq p_{0} .
$$

So that for all $x \in K$ (e.g., $v_{+}(x) e^{2 p} \geq b e^{2 p} \geq a e^{-2 p}+\frac{b}{2} e^{2 p} \geq v_{-}(x) e^{-2 p}+\frac{b}{2} e^{2 p} \geq$ $v_{-}(x) e^{-2 p}$ for $\left.p \geq p_{0}\right)$

$$
\begin{aligned}
& \left|\partial_{p} H(x, p)\right| \leq \begin{cases}2 e^{2 p} v_{+}(x) & \text { if } p \geq p_{0} \\
2 e^{-2 p} v_{-}(x) & \text { if } p \leq-p_{0}\end{cases} \\
& \left|\partial_{p} H(x, p)\right| \geq a e^{2|p|} \quad \text { if }|p| \geq p_{0} .
\end{aligned}
$$

Note that (78) also implies $\left|\partial_{p} H(x, p)\right| \geq 2$ for $|p| \geq p_{0}$ and thus that $\theta(r)=r \log r$ for $r=\left|\partial_{p} H(x, p)\right|$.

Using (76) and (77) we obtain the following lower bounds

$$
\begin{aligned}
& \left|p \partial_{p} H(x, p)-H(x, p)\right|-\theta\left(\left|\partial_{p} H(x, p)\right|\right) \\
& \quad \geq\left\{\begin{array}{cc}
\psi(p) v_{+}(x)-\frac{1}{2} e^{2 p} v_{+}(x) \log \left(2 e^{2 p} v_{+}(x)\right) & \text { if } p \geq p_{0}, \\
\geq a\left(\psi(p)-p e^{2 p}-\frac{1}{2} e^{2 p} \log (2 b)\right) & \text { if } p \leq-p_{0} . \\
\psi(-p) v_{-}(x)-\frac{1}{2} e^{-2 p} v_{-}(x) \log \left(2 e^{-2 p} v_{-}(x)\right) & \geq a\left(\psi(-p)+p e^{-p}-\frac{1}{2} e^{-2 p} \log (2 b)\right)
\end{array}\right.
\end{aligned}
$$

As $p \mapsto \psi(p)-p e^{2 p}-\frac{1}{2} e^{2 p} \log (2 b)$ is bounded from below for $p \in[0, \infty)$, this implies that there exists a $c>0$ such that the inequality in 73 holds for $p$ with $|p| \geq p_{0}$.

As (76) implies that $p \partial_{p} H(x, p)-H(x, p) \geq 0$ and as $\theta\left(\left|\partial_{p} H(x, p)\right|\right)$ is bounded from above for $x \in K$ and $|p| \leq p_{0}$, we can choose $c$ such that $(73)$ holds for all $p \in \mathbb{R}$.

By (75) we have $\left|\partial_{x} H(x, p)\right|+|p| \leq b e^{2|p|}+|p|$ for all $x \in K$ and $p \in \mathbb{R}$.

To conclude (iv), by (78) it is sufficient (and not difficult) to see that there exists a $c$ such that $b e^{2|p|}+|p| \leq c \frac{1}{2} e^{2|p|}(2|p|+\log (2 a))$ in case $|p| \geq p_{0}$ and $b e^{2|p|}+|p| \leq c$ in case $|p| \leq p_{0}($ as $\theta \geq 1)$.

Lemma A.3. Let $H$ satisfy Assumption 2.10(a) for the \pm 1 -space-model, then $H$ satisfies Assumption 4.10 . 
Proof. Assumption 4.1 has been verified in Lemma A.1. We consider $S$ with $S^{\prime}(x)=$ $\frac{1}{2} \log \frac{v_{-}(x)}{v_{+}(x)}$. Note that $\lim _{x \downarrow-1} S^{\prime}(x)=-\infty$ and $\lim _{x \uparrow 1} S^{\prime}(x)=\infty$ because of Assumption 2.3 (a). The integration constant of $S$ is chosen by choosing the infimum of $S$ equal to 0 . As $v_{+}, v_{-}$are twice-continuously differentiable and positive on the interior, also $S$ is twice continuously differentiable on the interior.

We leave the calculations for Assumptions 4.10 (a) and (b) for the reader (for (a) one computes that $\partial_{p} H(x, 0) S^{\prime}(x) \leq 0$ for all $\left.x \in(-1,1)\right)$.

By (b), $\partial_{p} H(x, p)=-\partial_{p} H\left(x, S^{\prime}(x)-p\right)$, thus $\partial_{p} H\left(x, \frac{1}{2} S^{\prime}(x)\right)=0$ and so

$$
\mathscr{L}(x, 0)=H\left(x, \frac{1}{2} S^{\prime}(x)\right) .
$$

With this we have

$$
\partial_{y} \mathscr{L}(y, 0)=\left(\frac{v_{+}^{\prime}(y)}{\sqrt{v_{+}(y)}}-\frac{v_{-}^{\prime}(y)}{\sqrt{v_{-}(y)}}\right)\left(\sqrt{v_{+}(y)}-\sqrt{v_{-}(y)}\right) .
$$

By Assumption 2.3 (a) and (c) it then follows that $\partial_{y} \mathscr{L}(y, 0)$ converges to $-\infty$ at -1 and to $\infty$ at 1 . This shows that (c) is satisfied.

For (d) we consider the -1 boundary, the other case follows similarly. By Cauchy's mean-value theorem, there exists $y \in(-1, x)$ such that

$$
\frac{\mathscr{L}(x, 0)-\mathscr{L}(-1,0)}{S(x)-S(-1)}=\frac{\partial_{y} \mathscr{L}(y, 0)}{\partial_{y} S(y)}
$$

Hence (using Cauchy's mean-value theorem)

$$
\begin{aligned}
\lim _{y \rightarrow-1} \frac{\partial_{y} \mathscr{L}(y, 0)}{\partial_{y} S(y)} & =v_{-}^{\prime}(-1) \sqrt{v_{+}(-1)} \lim _{y \rightarrow-1} \frac{-v_{-}(y)^{-\frac{1}{2}}}{\frac{1}{2} \log v_{-}(y)-\frac{1}{2} \log v_{+}(y)} \\
& =v_{-}^{\prime}(-1) \sqrt{v_{+}(-1)} \lim _{y \rightarrow-1} \frac{v_{-}^{\prime}(y) v_{-}(y)^{-\frac{3}{2}}}{v_{-}^{\prime}(y) v_{-}(y)^{-1}-v_{+}^{\prime}(y) v_{+}(x)^{-1}}=\infty
\end{aligned}
$$

\section{A.2 Verification for the Brownian example}

Lemma A.4. Let $H$ satisfy Assumption 2.10(a) for the $\mathbb{R}$-space-model, then $H$ satisfies Assumption 4.1 .

Proof. The proof follows the same lines as the proof of Lemma A.1 using $\theta(r)=$ $c \max \left\{|r|^{2}, 1\right\}$. The calculations in this setting are significantly easier.

Lemma A.5. Let $H$ satisfy Assumption 2.10(a) for the $\mathbb{R}$-space-model, then $H$ satisfies Assumption 4.11 .

Proof. By [CK17, Lemma 3.4], the one-sided Lipschitz property of $W^{\prime}$ implies that $\Upsilon(x)=\log \left(1+\frac{1}{2} x^{2}\right)$ is appropriate. 


\section{References}

[BC97] Martino Bardi and Italo Capuzzo-Dolcetta. Optimal control and viscosity solutions of Hamilton-Jacobi-Bellman equations. Boston, MA: Birkhäuser, 1997.

[CK17] Francesca Collet and Richard C. Kraaij. Dynamical moderate deviations for the Curie-Weiss model. Stochastic Processes and their Applications, 127(9):2900 $2925,2017$.

[Com89] F. Comets. Large deviation estimates for a conditional probability distribution. applications to random interaction Gibbs measures. Probability Theory and Related Fields, 80(3):407-432, 1989.

[CS04] Piermarco Cannarsa and Carlo Sinestrari. Semiconcave functions, HamiltonJacobi equations, and optimal control, volume 58. Springer Science \& Business Media, 2004.

[dHRvZ15] Frank den Hollander, Frank Redig, and Willem van Zuijlen. Gibbsnon-Gibbs dynamical transitions for mean-field interacting Brownian motions. Stochastic Processes and their Applications, 125(1):371 - 400, 2015.

[DPdH96] Paolo Dai Pra and Frank den Hollander. Mckean-Vlasov limit for interacting random processes in random media. Journal of Statistical Physics, 84(3-4):735$772,1996$.

[EK86] Stewart N. Ethier and Thomas G. Kurtz. Markov processes: Characterization and Convergence. Wiley, 1986.

[EK10] Victor Ermolaev and Christof Külske. Low-temperature dynamics of the CurieWeiss model: Periodic orbits, multiple histories, and loss of Gibbsianness. Journal of Statistical Physics, 141(5):727-756, 2010.

[FdHM13] R. Fernández, F. den Hollander, and J. Martínez. Variational description of Gibbs-non-Gibbs dynamical transitions for the Curie-Weiss model. Communications in Mathematical Physics, 319(3):703-730, 2013.

[FK06] Jin Feng and Thomas G. Kurtz. Large Deviations for Stochastic Processes. American Mathematical Society, 2006.

[Fle69] Wendell H. Fleming. The Cauchy problem for a nonlinear first order partial differential equation. J. Differential Equations, 5:515-530, 1969.

[FS89] W. H. Fleming and H. M. Soner. Asymptotic expansions for Markov processes with Lévy generators. Applied Mathematics and Optimization, 19(1):203-223, 1989.

[FS06] Wendell H. Fleming and H. Mete Soner. Controlled Markov processes and viscosity solutions. 2nd ed. New York, NY: Springer, 2nd ed. edition, 2006. 
[FW98] M.I. Freidlin and A.D. Wentzell. Random perturbations of dynamical systems. Springer-Verlag, second edition, 1998.

[GHR03] Misha Guysinsky, Boris Hasselblatt, and Victoria Rayskin. Differentiability of the Hartman-Grobman linearization. Discrete and Continuous Dynamical Systems, 9(4):979-984, 2003.

[KK19] Sascha Kissel and Christof Külske. Dynamical Gibbs-non-Gibbs transitions in Curie-Weiss Widom-Rowlinson models. Markov Process. Related Fields, 25(3):379-413, 2019.

[KLN07] Christof Külske and Arnaud Le Ny. Spin-flip dynamics of the Curie-Weiss model: Loss of Gibbsianness with possibly broken symmetry. Communications in Mathematical Physics, 271(2):431-454, 2007.

[KM20] Christof Külske and Daniel Meißner. Dynamical Gibbs-non-Gibbs transitions in the Curie-Weiss Potts model in the regime beta< 3. preprint; ArXiv:2011.00350, 2020 .

[Kra16a] Richard C. Kraaij. A large deviation perspective on exponential decay of entropy and lower bounds on the Ricci-curvature. preprint; ArXiv:1609.09637, 2016.

[Kra16b] Richard C. Kraaij. Large deviations for finite state Markov jump processes with mean-field interaction via the comparison principle for an associated HamiltonJacobi equation. Journal of Statistical Physics, 164(2):321-345, 2016.

[Kra16c] Richard C. Kraaij. Semigroup methods for large deviations of Markov processes. PhD thesis, Delft University of Technology, 2016.

[Léo95] Christian Léonard. Large deviations for long range interacting particle systems with jumps. Annales de l'institut Henri Poincaré (B) Probabilités et Statistiques, 31(2):289-323, 1995.

[Lio82] Pierre-Louis Lions. Generalized solutions of Hamilton-Jacobi equations, volume 69 of Research Notes in Mathematics. Pitman (Advanced Publishing Program), Boston, Mass.-London, 1982.

[LY95] Xun Jing Li and Jiong Min Yong. Optimal control theory for infinite-dimensional systems. Systems \& Control: Foundations \& Applications. Birkhäuser Boston, Inc., Boston, 1995.

[Per01] Lawrence Perko. Differential equations and dynamical systems, volume 7 of Texts in Applied Mathematics. Springer-Verlag, New York, third edition, 2001.

[PR14] Etienne Pardoux and Aurel Răşcanu. Stochastic differential equations, backward SDEs, partial differential equations, volume 69 of Stochastic Modelling and Applied Probability. Springer, Cham, 2014. 
[RAS15] Firas Rassoul-Agha and Timo Seppäläinen. A course on large deviations with an introduction to Gibbs measures, volume 162 of Graduate Studies in Mathematics. American Mathematical Society, Providence, RI, 2015.

[RS82] A. C. M. van Rooij and W. H. Schikhof. A second course on real functions. Cambridge University Press, Cambridge-New York, 1982.

[Run05] Volker Runde. A taste of topology. Universitext. Springer, New York, 2005.

[RW12] Frank Redig and Feijia Wang. Gibbs-non-Gibbs transitions via large deviations: Computable examples. Journal of Statistical Physics, 147(6):1094-1112, 2012.

[Tes12] Gerald Teschl. Ordinary differential equations and dynamical systems, volume 140 of Graduate Studies in Mathematics. American Mathematical Society, Providence, RI, 2012.

[vEFdHR02] A.C.D. van Enter, R. Fernández, F. den Hollander, and F. Redig. Possible loss and recovery of Gibbsianness during the stochastic evolution of Gibbs measures. Communications in Mathematical Physics, 226:101-130, 2002.

[vEFdHR10] A.C.D. van Enter, R. Fernández, F. den Hollander, and F. Redig. A largedeviation view on dynamical Gibbs-non-Gibbs transitions. Moscow Mathematical Journal, 10:687-711, 2010.

[vZ16] W. B. van Zuijlen. Gibbs-non-Gibbs Transitions and Vector-Valued Integration. PhD thesis, Leiden University, 2016. 